\title{
The $5 \alpha$-reductase inhibitor finasteride reduces opioid self-administration in animal models of opioid use disorder
}

\author{
Gabriel D. Bosse, ${ }^{1}$ Roberto Cadeddu, ${ }^{1}$ Gabriele Floris, ${ }^{1}$ Ryan D. Farero, ${ }^{2}$ Eva Vigato, ${ }^{1}$ Suhjung J. Lee, ${ }^{2}$ Tejia Zhang, ${ }^{1}$ \\ Nilesh W. Gaikwad, ${ }^{3}$ Kristen A. Keefe, ${ }^{1}$ Paul E.M. Phillips, ${ }^{2}$ Marco Bortolato, ${ }^{1}$ and Randall T. Peterson ${ }^{1}$ \\ 'Department of Pharmacology and Toxicology, College of Pharmacy, University of Utah, Salt Lake City, Utah, USA. ²Department of Psychiatry and Behavioral Sciences, University of Washington, Seattle, \\ Washington, USA. ${ }^{3}$ Gaikwad Steroidomics Laboratory, Davis, California, USA.
}

\begin{abstract}
Opioid use disorder (OUD) has become a leading cause of death in the United States, yet current therapeutic strategies remain highly inadequate. To identify potential treatments for OUD, we screened a targeted selection of over 100 drugs using a recently developed opioid self-administration assay in zebrafish. This paradigm showed that finasteride, a steroidogenesis inhibitor approved for the treatment of benign prostatic hyperplasia and androgenetic alopecia, reduced self-administration of multiple opioids without affecting locomotion or feeding behavior. These findings were confirmed in rats; furthermore, finasteride reduced the physical signs associated with opioid withdrawal. In rat models of neuropathic pain, finasteride did not alter the antinociceptive effect of opioids and reduced withdrawal-induced hyperalgesia. Steroidomic analyses of the brains of fish treated with finasteride revealed a significant increase in dehydroepiandrosterone sulfate (DHEAS). Treatment with precursors of DHEAS reduced opioid self-administration in zebrafish in a fashion akin to the effects of finasteride. These results highlight the importance of steroidogenic pathways as a rich source of therapeutic targets for OUD and point to the potential of finasteride as a new treatment option for this disorder.
\end{abstract}

\section{Introduction}

Over the last decade, the widespread abuse of prescription painkillers, such as oxycodone and hydrocodone, has led to a crisis of opioid use disorder (OUD) and a dramatic increase in opioid overdoses in North America (1). The extent of this crisis is such that opioids account for more than $60 \%$ of all drug overdoses in the United States, with an estimated 47,000 to 50,000 fatalities annually. Synthetic opioids, such as fentanyl, are the major drivers of opioid overdoses $(1,2)$. Unfortunately, current therapeutic options for OUD are highly unsatisfactory. Existing treatments rely on replacement with long-acting opioids, such as methadone or buprenorphine (3). Although these options help patients cope with drug craving and manage withdrawal symptoms (3), they are not ideal because of their intrinsic liability for abuse and dependence (4). This background highlights the urgent need for improved therapeutic options to reduce the risk and severity of OUD, particularly in patients requiring opioid treatment for chronic and neuropathic pain syndromes (for which effective alternatives to these painkillers are not always available).

Rodent models have been successfully used to model substance use disorders and study the circuitry and neurobiological changes involved in drug abuse $(5,6)$. However, the viability of

Authorship note: RC and GF contributed equally to this work.

Conflict of interest: The authors have declared that no conflict of interest exists. Copyright: (5) 2021, American Society for Clinical Investigation.

Submitted: September 8, 2020; Accepted: April 8, 2021; Published: May 17, 2021.

Reference information: J Clin Invest. 2021;131(10):e143990.

https://doi.org/10.1172/JCl143990. these models to screen for more effective therapeutic candidates is limited by their low throughput and high costs. In recent years, the zebrafish (Danio rerio) has emerged as a new alternative to study a wide range of complex behavioral and neuropsychiatric disorders, such as schizophrenia and depression (7-9). Importantly, zebrafish have been shown to develop conditioned place preference and withdrawal symptoms after exposure to opioids and other drugs of abuse, such as cocaine and alcohol (10-14). Additionally, adult zebrafish possess a complex central nervous system, including a blood-brain barrier, and considerable similarities with the mammalian homolog. Furthermore, this model is well suited for the rapid testing of candidate drugs because compounds of interest can be dissolved directly into the water of the tank (15-17). Therefore, zebrafish models offer a unique opportunity to combine drug-discovery screening and substance abuse research.

Using a recently described paradigm to condition adult fish to self-administer the opioid hydrocodone (18), we designed a behavior-based screen to identify compounds affecting opioid self-administration in zebrafish. We screened 110 unique molecules selected for their annotated activity against processes and pathways known to be involved in substance abuse disorders. From this screen, we identified the $5 \alpha$-reductase $(5 \alpha \mathrm{R})$ inhibitor finasteride $(19,20)$ as one of the most effective compounds to reduce opioid self-administration without affecting food seeking or overall locomotion. $5 \alpha \mathrm{R}$ catalyzes the rate-limiting step of the conversion of several ketosteroids, including progesterone and testosterone, into their neuroactive metabolites dihydroprogesterone and dihydrotestosterone (DHT). In turn, these steroids are further converted into the neurosteroids allopregnanolone 
A

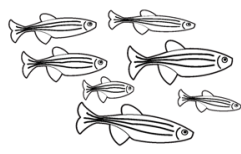

Conditioned animals 15 animals/group

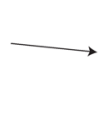

$$
\begin{gathered}
100 \text { compounds } \\
10 \mu \mathrm{M}
\end{gathered}
$$$$
60 \text { minutes }
$$
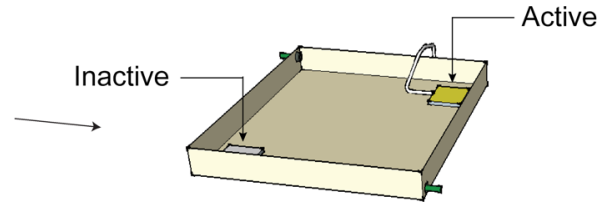

30 minutes

self-administration assay
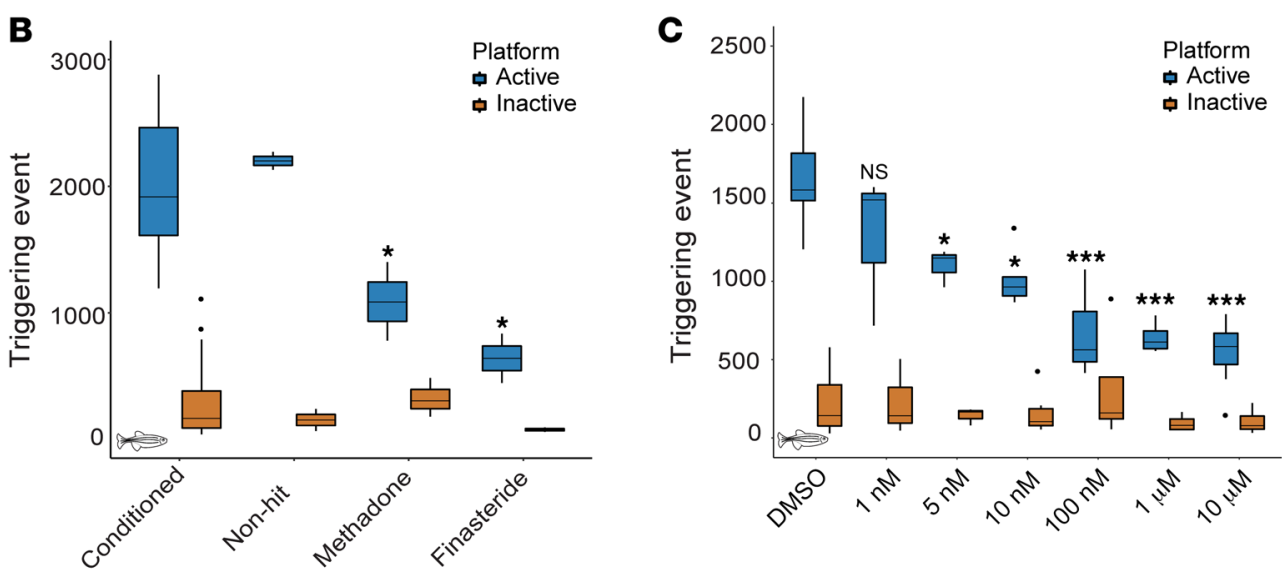

Figure 1. Small-molecule screen for modulators of opioid self-administration. (A) Conditioned animals were treated with the different molecules at $10 \mu \mathrm{M}$ for 60 minutes before assessing their opioid self-administration for 30 minutes. (B) Known small molecules affect opioid self-administration. Treatment with methadone $(n=$ $3)$ and finasteride $(n=2)$ significantly reduced the number of triggering events at the active platform. Conditioned fish: $n=56$. No difference was observed at the inactive platform. $P$ value calculated with Student's $t$ test compared with conditioned animals. (C) Dose-response experiment for finasteride. Three doses, $100 \mathrm{nM}, 1 \mu \mathrm{M}$, and $10 \mu \mathrm{M}$, reduced the number of triggering events below our threshold of 1000 activations. $P$ value computed by Tukey's honestly significant difference test on 1-way ANOVA, inactive platform $[F(6,36)=1.06, P=0.40]$ and active platform $\left[F(6,36)=24.60, P=2.31 \times 10^{-11}\right]$ compared with the DMSO control. No significant difference was detected for the inactive platform. DMSO: $n=16,1 \mathrm{nM}: n=3,5 \mathrm{nM}: n=5,10 \mathrm{nM}: n=6,100 \mathrm{nM}: n=5,1 \mu \mathrm{M}: n=4$, $10 \mu \mathrm{M}: n=7 .{ }^{*} P<0.05,{ }^{* *} P<0.01,{ }^{* * *} P<1 \times 10^{-5}$. Each $n$ value represents a group of 15 animals. These experiments were performed using a between-subjects design. All box plots were generated using $\mathrm{R}$ graphic programming and the ggplot module. The lower and upper hinges correspond to the first and third quartiles. The line indicates the median. The whiskers extend from the hinges to the maximum or minimum value at most 1.5 times the IQR from the hinge. Data points beyond that are considered outliers.

ed in the delivery of hydrocodone directly at the platform. We used 15 animals per group of fish, so each $n$ represents a unique group of 15 fish. Potential modulators of opioid self-administration were tested by training fish to self-administer hydrocodone for 4 days and treating with the compound of interest on the fifth day (Figure 1A). Before performing the small-molecule screen, we sought to validate the screening method by testing one of the only treatments used in the clinic for OUD, the slow-acting opioid methadone. Fish conditioned for 4 days to self-administer hydrocodone were treated with methadone $(1 \mathrm{mg} / \mathrm{L})$ for 60 minutes and then transferred to the self-administration arena. During each 30-minute self-administration session, the number of triggering events for the active and inactive platforms was recorded and used as a readout of opioid intake. Methadone significantly reduced hydrocodone self-administration (Figure 1B), suggesting that the screening assay can identify molecules that reduce opioid selfadministration.

Small-molecule screening identifies modulators of opioid selfadministration. We conducted a small-scale screen using a tar-

and $3 \alpha$-androstanediol (3a-diol, refs. 21-24), which play an essential role in behavioral regulation. Finasteride has been clinically approved for over 25 years as a treatment for benign prostatic hyperplasia and male-pattern baldness (25). These effects reflect the suppression of DHT synthesis.

The effects on self-administration were also confirmed in rats. Notably, we found that finasteride opposed the physical effects and hyperalgesia associated with opioid withdrawal but did not reduce the pain-killing properties of opioids in rat models of neuropathic pain. Finally, we identified the neurosteroid dehydroepiandrosterone sulfate (DHEAS) as a likely mediator of finasteride's effects. We thus have uncovered a role for neuroactive steroids in the control of opioid self-administration.

\section{Results}

Validation of the screening method. To screen for modulators of opioid self-administration, we utilized our newly developed assay to condition fish with the opioid hydrocodone. As previously described, small groups of adult zebrafish were conditioned to swim across an active platform to receive a dose of the drug (18). Each visit result- geted collection of compounds selected based on hypotheses presented in the literature regarding neuronal pathways contributing to addiction, their ability to modulate pathways identified in GWAS studies on substance abuse patients, and molecular pathways affected during substance abuse. We hypothesized that focusing on this targeted collection would increase the probability of finding effective drugs within a smaller library of compounds. To test whether the compounds reduce opioid self-administration, drug-conditioned animals were treated with $10 \mu \mathrm{M}$ of each candidate compound 60 minutes before the 30-minute self-administration session (Figure 1A). On average, control animals triggered the release of drug more than 1800 times per session. To reduce the number of false-positive hits, we tested each compound in duplicate; furthermore, only compounds with fewer than 1000 triggering events for both duplicates were considered hits.

Finasteride reduces opioid self-administration. After screening over 100 compounds, we identified the $5 \alpha \mathrm{R}$ inhibitor finasteride as highly effective in reducing opioid self-administration. Incubation with a single dose of $10 \mu \mathrm{M}$ of finasteride for 60 minutes was sufficient to reduce the number of triggering events at the 

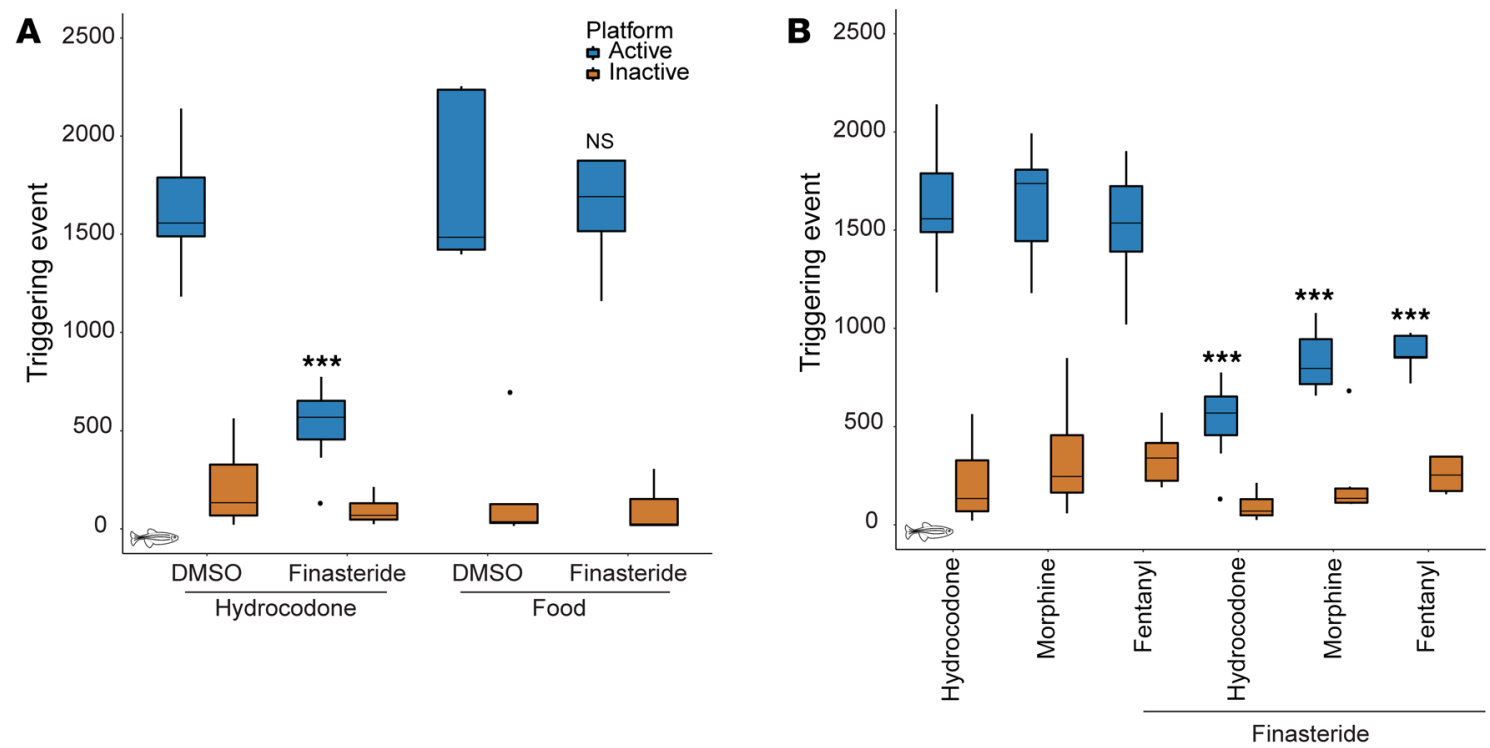

Figure 2. Finasteride effectively reduces the self-administration of different opioids but does not affect all motivated behaviors. (A) Finasteride reduced opioid self-administration without affecting food-seeking behavior. Finasteride significantly reduced the number of triggering events at the active platform compared with DMSO control for hydrocodone. No difference was detected for fish conditioned to self-administer food. $P$ value computed by Tukey's honestly significant difference (HSD) test on 1-way ANOVA; inactive platform $[F(3,29)=1.05, P=0.38]$ and active platform $[F(3,29)=19.37, P=$ $4.32 \times 10^{-7}$ compared with respective DMSO control. Opioid-trained animals - DMSO: $n=16$, finasteride: $n=7$. Food seeking - DMSO: $n=5$, finasteride: $n$ $=6$. These experiments were performed using a between-subject design. (B) Finasteride affects opioid self-administration for animals conditioned with 3 different opioids. $P$ value computed by Tukey's HSD test on 1-way ANOVA; inactive platform $[F(5,81)=1.57, P=0.18]$ and active platform $[F(5,81)=31.68$, $P=4.52 \times 10^{-13}$ compared with respective control. Hydrocodone control: $n=16$, hydrocodone plus finasteride: $n=7$, morphine control: $n=7$, morphine plus finasteride: $n=6$, fentanyl control: $n=7$, fentanyl plus finasteride: $n=5$. No significant difference was detected for the inactive platform in any condition. ${ }^{*} P<0.05,{ }^{* *} P<0.01,{ }^{* *} P<1 \times 10^{-5}$. Each $n$ value represents a group of 15 animals. Data for hydrocodone treatment alone reproduced from Figure $1 \mathrm{C}$ for comparison. These experiments were performed using a within-subject design. All box plots were generated using R graphic programming and the ggplot module. The lower and upper hinges correspond to the first and third quartiles. The line indicates the median. The whiskers extend from the hinges to the maximum or minimum value at most 1.5 times the IQR from the hinge. Data points beyond that are considered outliers.

active platform by $73 \%$ (Figure 1B). The complete list of tested compounds and their effects on opioid self-administration is presented in Supplemental Figure 1 and Supplemental Table 1; supplemental material available online with this article; https://doi. org/10.1172/JCI143990DS1.

To further validate that the inhibition of $5 \alpha \mathrm{R}$ was responsible for reducing opioid self-administration, we tested a different $5 \alpha \mathrm{R}$ inhibitor, dutasteride (26). Like finasteride, dutasteride reduced the number of triggering events at the active platform (Supplemental Figure 2). Although research on neuroactive steroids in zebrafish has been limited, the key steroidogenic enzymes are expressed in the adult brain, and the activity of $5 \alpha \mathrm{R}$ has been detected in brain extracts (27-33). Additionally, using a publicly available single-cell RNA-Seq library, we were able to detect $5 \alpha$ R transcripts in several different cell types in zebrafish brains (ref. 34 and Supplemental Figure 3). Taken together, these results suggest that the inhibition of the enzyme $5 \alpha$ R reduced opioid self-administration in zebrafish.

To further characterize the efficacy of finasteride, we performed a dose-response experiment. We used 10-fold dilutions to test finasteride concentrations from $10 \mu \mathrm{M}$ to $1 \mathrm{nM}$. A significant difference was detected with concentrations as low as $5 \mathrm{nM}$ (Figure 1C). This dose-response experiment supports the idea that finasteride is highly potent and has a large therapeutic window in zebrafish.

Finasteride does not affect locomotion or food self-administration. To determine whether the effect of finasteride was caused by sedation, we monitored the swimming speed of finasteride-treated animals. Finasteride did not reduce locomotion in comparison with DMSO (Supplemental Figure 4). Importantly, we also did not observe any significant difference in the number of triggering events at the inactive platform between the finasteride-treated fish and control animals (Figure 1). These results suggest that finasteride specifically reduced the number of triggering events at the active platform without affecting overall locomotion.

Drugs of abuse are known to activate the reward pathway in the brain, which is a core contributor to motivated behaviors, such as feeding or reproduction (35). We thus decided to test whether finasteride was also affecting other motivated behaviors by measuring food self-administration in zebrafish. We first trained fish as previously reported for opioids but used food instead of opioid as the reward. Food-conditioned animals were then treated with DMSO or finasteride for 60 minutes, and the number of triggering events at the active platform was measured. As opposed to hydrocodone self-administration, finasteride-treated animals exhibited no decrease in food seeking (Figure 2A). These data suggest that finasteride did not affect all motivated behaviors and further validate that this drug did not impair locomotion.

Finasteride reduces self-administration of different opioids. It has been described that each class of opioids has a different abuse potential $(36,37)$. Therefore, we decided to test the effect of finasteride on animals conditioned with the traditional opioid most 
A
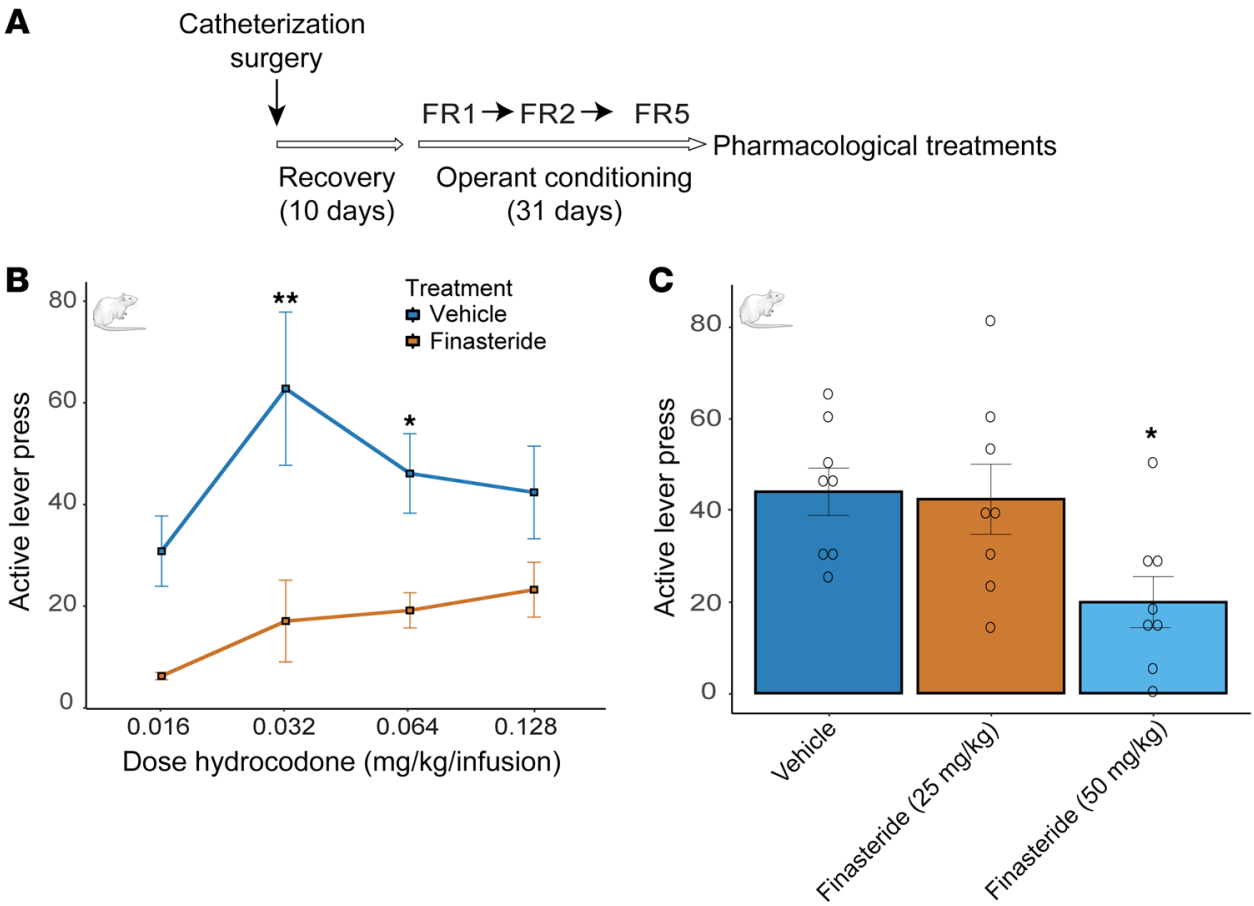

Figure 3. Finasteride treatment reduces opioid self-administration in rats. (A) Rats were conditioned to self-administer hydrocodone and after establishing robust FR5, they were treated with either vehicle or finasteride. (B) A total of 6 animals per condition were tested with active lever presses for animals trained with different concentrations of hydrocodone. Treatment with finasteride $(50 \mathrm{mg} / \mathrm{kg})$ reduced opioid selfadministration of hydrocodone for animals conditioned with 0.032 and $0.064 \mathrm{mg} / \mathrm{kg}$. $P$ value was corrected for multiple-comparison 2-way ANOVA $[F(1,38)=30.5, P=0.0001]$. $P$ value compared finasteride-treated versus vehicle-treated animals ${ }^{*} P<0.05,{ }^{* *} P<0.01$. (C) Injection (i.p.) with $50 \mathrm{mg} / \mathrm{kg}$ but not $25 \mathrm{mg} / \mathrm{kg}$ finasteride reduced the number of active lever presses for $0.064 \mathrm{mg} / \mathrm{kg}$ hydrocodone; 8 animals were treated for each dose of finasteride. $P$ values were corrected for multiple comparisons by 1-way ANOVA $[F(2,21)=4.69$, $P=0.02]$. Data are shown as the mean \pm SEM. Adult male Sprague-Dawley rats were used to perform the hydrocodone self-administration assay. These experiments were performed using a within-subjects design.

oid consumption. To confirm this effect in mammals, we tested finasteride in a rat model of hydrocodone self-administration. Adult male Sprague-Dawley rats were first conditioned to press a lever to receive an i.v. infusion of hydrocodone (0.016-0.128 mg/ $\mathrm{kg} / 160 \mu \mathrm{L})$. Operant conditioning consisted of 3 stages of fixedratio (FR) reinforcement schedules: FR1, FR2, and FR5 (i.e., FR1 = each lever press resulted in a hydrocodone infusion). Animals progressed to the next stage of conditioning after reaching the criterion of more than $70 \%$ of lever presses being on the lever on the previous schedule (Figure 3A and Supplemental Figure 5). Importantly, to mimic the zebrafish conditions, rats had access to the drug for 60 minutes per day. To find the optimal concentration for hydrocodone conditioning, we tested a range of doses from $0.016 \mathrm{mg} /$ $\mathrm{kg}$ to $0.128 \mathrm{mg} / \mathrm{kg}$. We found that animals conditioned with $0.032 \mathrm{mg} /$ $\mathrm{kg}$ and $0.064 \mathrm{mg} / \mathrm{kg}$ had the highest number of lever presses after 31 days of training (Figure 3B). We then treated animals conditioned with each concentration of hydrocodone by i.p. injection of finasteride (50 $\mathrm{mg} / \mathrm{kg}$ ) or vehicle before a self-ad-

commonly used in animal models, morphine, and one of the most potent and deadly opioids, the synthetic opioid fentanyl.

In order to test the effect of finasteride, we used the same conditioning protocol to train animals to self-administer morphine and fentanyl. Given that morphine and hydrocodone are closely related, we used the same dose of $6 \mathrm{mg} / \mathrm{L}$. As for fentanyl, studies suggest that it is up to $50-100$ times more potent than morphine (38), so a dose 50 times lower $(0.12 \mathrm{mg} / \mathrm{L})$ was used to take into account its greater potency. We first confirmed that animals conditioned with either opioid had similar self-administration levels after 5 days of conditioning. There was no significant difference in the number of triggering events between fish trained with different opioids (Figure 2B). Therefore, this conditioning protocol is easily applicable to multiple classes of opioids. The conditioned animals were then treated with $10 \mu \mathrm{M}$ finasteride for 60 minutes before performing a 30-minute self-administration session. As observed with hydrocodone-trained animals, there was a reduction in the number of visits to the active platform without affecting the inactive platform (Figure $2 \mathrm{~B}$ ). This result suggests that finasteride reduced opioid self-administration behavior regardless of the opioid used during the conditioning phase.

Finasteride reduces opioid self-administration in rats. The zebrafish model suggests that finasteride strongly reduces opi- ministration session. That dose of finasteride was sufficient to significantly reduce the number of active lever presses at both 0.032 and $0.064 \mathrm{mg} / \mathrm{kg} /$ infusion of hydrocodone (Figure $3 \mathrm{~B}$ ).

To determine whether finasteride was effective at different doses, we treated animals conditioned with $0.064 \mathrm{mg} / \mathrm{kg}$ of hydrocodone with $25 \mathrm{mg} / \mathrm{kg}$ or $50 \mathrm{mg} / \mathrm{kg}$ of finasteride. We confirmed that injection of $50 \mathrm{mg} / \mathrm{kg}$ significantly reduced the number of active lever presses, but there was no difference in animals treated with the lower dose (Figure 3C). Importantly, locomotion or inactive lever presses were not affected by finasteride (Supplemental Figures 6 and 7).

To further validate that finasteride can reduce opioid intake in mammals, we also tested the effect of finasteride on the potent synthetic opioid fentanyl. Adult male and adult female Wistar rats were used to perform the fentanyl self-administration assay. Rats were trained to perform a nose-poke to trigger the release of a drop of fentanyl drinking solution $(0.02 \mathrm{mg} / \mathrm{kg} /$ delivery) in a liquid magazine tray. We trained both male and female animals in 15 sessions of 60 minutes. As the training progressed, we observed an increase in active nose-pokes per session, and animals rapidly learned to discriminate between the active and inactive nosepoke holes (Figure 4A). After each self-administration session, the fentanyl consumed $(\mathrm{mg} / \mathrm{kg})$ was calculated for each animal by 

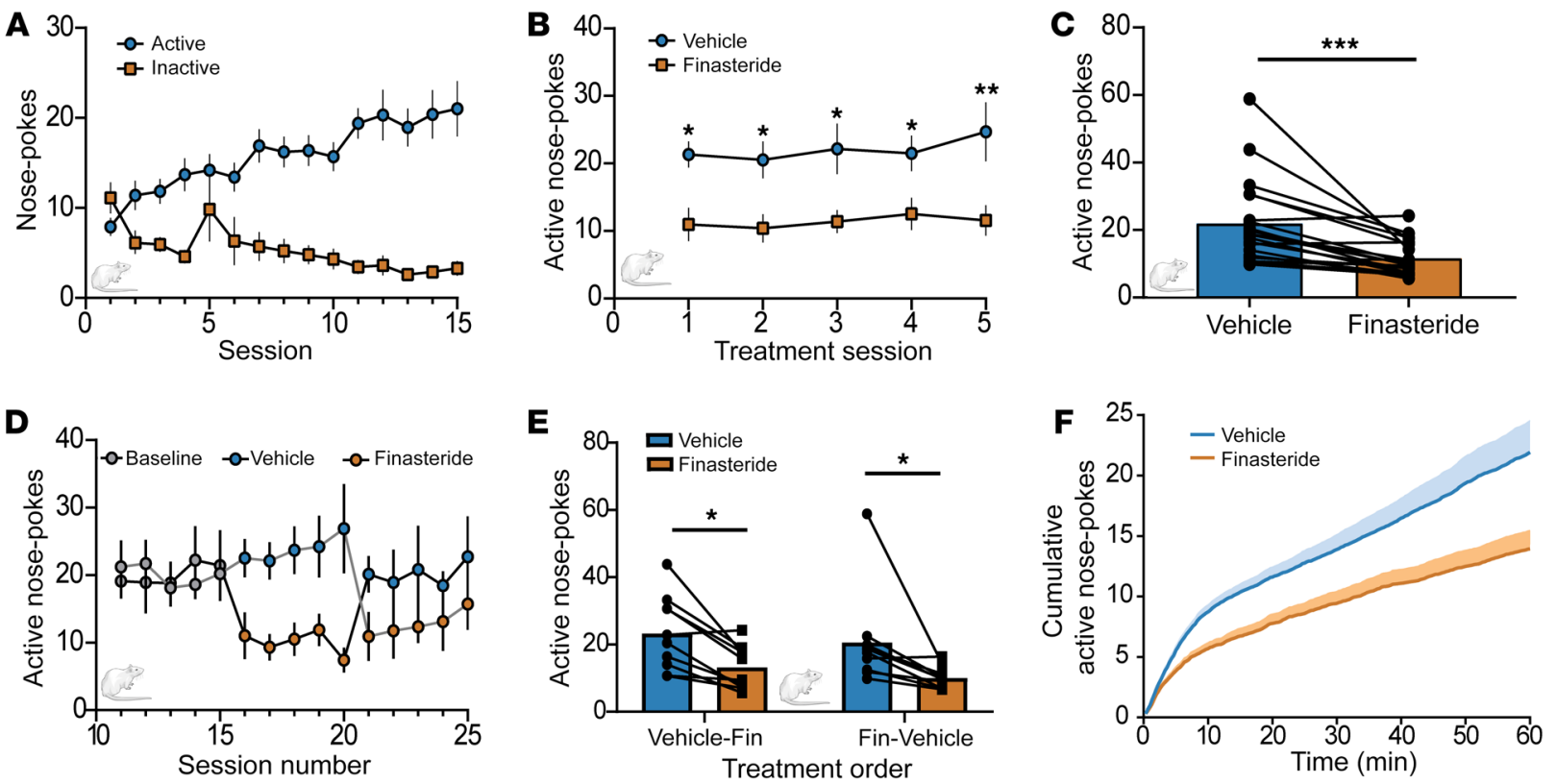

Figure 4. Finasteride decreases operant response for oral fentanyl self-administration. (A) Operant nose-poke responses at the active (blue circles) and inactive (orange squares) nose-poke ports during baseline fentanyl self-administration sessions ( $n=20$ rats). (B) Daily finasteride injections (i.p., 50 mg/ $\mathrm{kg}$ ) (orange squares) decreased active nose-pokes compared with vehicle injections (blue circles). A main effect of drug treatment was observed. Sidak's post hoc analysis was performed for multiple comparisons on a mixed-effect model $[F(1,19)=20.77, P=0.0002]$. (C) Average operant responses during vehicle (blue) and finasteride (orange) treatment for each individual animal. A paired $t$ test revealed finasteride significantly decreased the animals' active response for fentanyl delivery $[t(19)=4.481, P=0.0003]$. ( $\mathbf{D}$ and $\mathbf{E}$ ) Animals received daily injections of finasteride during sessions $16-20$ ( $n=10$ rats, $\mathbf{D}$ : black line, E: finasteride-vehicle) or during sessions $21-25$ ( $n=10$ rats, $\mathbf{D}$ : gray line, E: vehicle-finasteride). (D) The baseline refers to animals responding during sessions 10-15. (E) The order of treatment had no interaction with the effect of the treatment $[F(1,18)=0.007, P=0.93]$. A main effect of treatment was observed $[F(1,18)=19.03, P=0.0004]$. Sidak's analysis was performed for multiple comparisons on 2-way ANOVA. (F) Average cumulative plot of active nose-poke responses during the vehicle-treated (blue) or finasteride-treated (orange) sessions $(n=20)$. ${ }^{*} P<0.05,{ }^{*} P<0.01$, ${ }^{* * *} P<0.001$. Data are shown as the mean \pm SEM. Adult male and adult female Wistar rats were used to perform the fentanyl self-administration assay. These experiments were performed using a within-subjects design.

subtracting the amount of fentanyl left in the magazine tray from the total amount of drug delivered. As observed for the number of active nose-pokes, we also detected an increase in ingested fentanyl over time (Supplemental Figure 8A).

After the conditioning phase, animals were separated into 2 groups, and the effect of finasteride was tested in 10 sessions. Each group was treated with either an i.p. injection of finasteride $(50 \mathrm{mg} / \mathrm{kg})$ or vehicle for 5 out of the 10 sessions. Treatments were then inversed for the remaining 5 sessions so that both groups received 5 sessions of finasteride and 5 of vehicle. As observed for hydrocodone self-administration, acute treatment with finasteride significantly reduced the number of active nose-pokes (Figure $4, \mathrm{~B}$ and C), as well as the total amount of fentanyl consumed per session (Supplemental Figure 8, B-E), without affecting the number of inactive nose-pokes (Supplemental Figure 9). This acute treatment with finasteride was effective during the 5 days of treatment, regardless of the order of treatment (Figure 4, D and E). Importantly, finasteride reduced opioid consumption in male and female animals (Supplemental Figure 10).

We also quantified the number of active nose-pokes over time during each session. In vehicle-treated animals, there was a rapid succession of active nose-pokes in the first 10 minutes before slowing down over the next 50 minutes. Finasteride-treated animals followed a similar pattern, but they only acquired approximately half of the nose-pokes before slowing their intake rate (Figure 4F).
These results collectively demonstrated that the activity of finasteride on opioid self-administration is conserved in mammals, even for animals conditioned with the synthetic opioid fentanyl.

Finasteride reduces the physical effects of opioid withdrawal. Given that previous studies have shown that neurosteroids play a role in shaping opioid withdrawal $(39,40)$, we tested whether finasteride may also modify the effects of opioid withdrawal using the model of naloxone-precipitated withdrawal syndrome in adult Long Evans male rats. Finasteride significantly reduced the number of wet-dog shakes and the duration of grooming behavior induced by naloxone (Figure 5); however, it did not fully reverse the jumping and digging responses induced by the opioid antagonist (Supplemental Figure 11). These results indicate that finasteride may reduce the severity of opioid withdrawal.

Finasteride does not affect the antinociceptive properties of opioids. Despite their high abuse potential, opioids are still invaluable as analgesics. In the absence of an effective alternative, they are still an essential treatment, especially for people with chronic pain. Therefore, an ideal candidate for an opioid abuse treatment would be a drug that does not affect the pain-killing properties of opioids. A previous report demonstrated that finasteride did not reduce the efficiency of morphine; however, the authors used a lower dose of finasteride $(25 \mathrm{mg} / \mathrm{kg})$ and tested a single nociceptive stimulus (40). To further validate that finasteride does not interfere with the antinociceptive effects of opioids in our condi- 
A

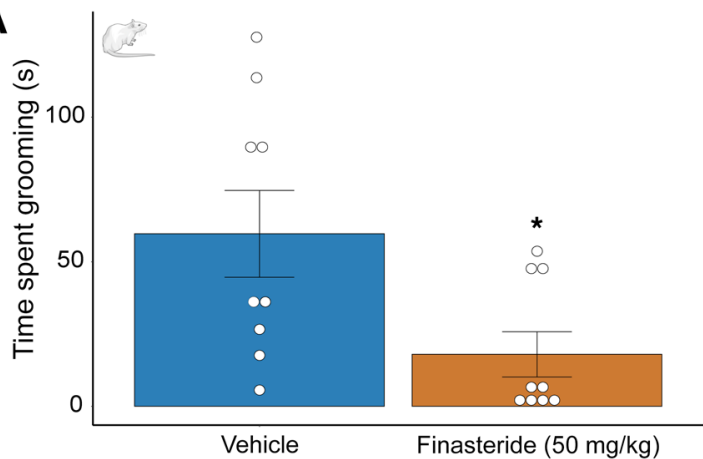

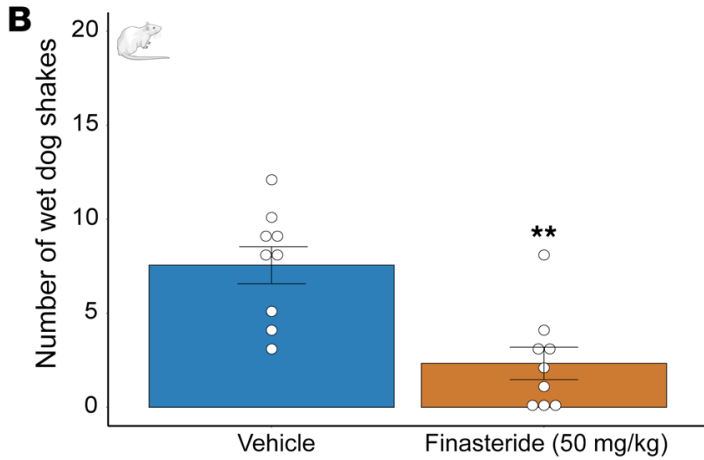

Figure 5. Finasteride reduces the physical effects of opioid withdrawal. Animals were treated with escalating doses of morphine for 5 days. On day 6 , rats received an acute dose of morphine ( $40 \mathrm{mg} / \mathrm{kg}$, s.c.) and after 1 hour, 40 minutes, received a second injection of either finasteride (50 mg/ $\mathrm{kg}$, i.p.) or vehicle. The opioid receptor antagonist naloxone $(1.5 \mathrm{mg} / \mathrm{kg}$, i.p.) was administered 20 minutes after the second injection with finasteride (or its vehicle). Behavioral testing started immediately after naloxone injection; animals were placed in a Plexiglass chamber, and their behavior was observed and recorded for 30 minutes. (A) In rats that were finasteride treated, naloxone-precipitated withdrawal was less severe, as indicated by less time spent grooming (observed in the finasteride-treated group). (B) Acute injection of finasteride reduced the number of wet-dog shakes in animals in morphine withdrawal. ${ }^{*} P<0.05,{ }^{* *} P<0.01$. Data are shown as the mean \pm SEM. $n=9$ for both conditions. $P$ values were calculated using an unpaired $t$ test with Welch's correction between finasteride- and vehicle-treated animals. Adult male Long-Evans rats were used to test naloxone-induced withdrawal. This experiment was performed with a between-subjects design and blind analysis.

tions, we used the most rigorous test available in rats by testing the impact of opioids on a rat model of neuropathy.

We performed spinal nerve ligation (SNL) surgery on adult male Sprague-Dawley rats (41); 14 days after surgery mechanical allodynia and nociception were measured by using the von Frey hair and Randall-Selitto tests, respectively $(42,43)$. Rats were separated into experimental groups and assigned to treatment based on their mechanical thresholds, to have identical or similar baselines (pretreatment). We also measured thermal nociception (hot plate) (44). We first validated the antinociceptive effects of opioids by testing different doses of morphine (1-3 mg/kg, s.c.) or hydrocodone (1-10 mg/kg, s.c.) every 30 minutes for 3 hours. For the Randall-Selitto test, an increase in mechanical force was applied to the paw until a withdrawal response was observed (Figure 6). Treatment with either morphine or hydrocodone significantly increased the force needed to trigger withdrawal compared with the same animals prior to treatment with the opioid (Figure 6A and Supplemental Figure 12B). We then selected the most effective dose for both opioids, morphine $(3 \mathrm{mg} / \mathrm{kg})$ and hydrocodone $(10 \mathrm{mg} / \mathrm{kg})$, and tested the effect of coinjection with finasteride $(50 \mathrm{mg} / \mathrm{kg}$, i.p.). The cotreatment with finasteride did not reduce the antinociceptive effect of either opioid (Figure 6B and Supplemental Figure 12C). We also performed the same set of experiments to test a different mechanical stimulus, i.e., the von Frey test. As with the Randall-Selitto test, we did not detect any reduction in the antinociceptive effects of the opioids by treatment of the rats with finasteride (Supplemental Figure 13).

In order to test a different type of nociception, we used the hot-plate assay to test for a thermal stimulus on a different group of neuropathic rats. Animals were placed on a hot-plate analgesia meter and the latency to lick their left hind paw was measured at different temperatures $\left(48.5^{\circ} \mathrm{C}\right.$ and $\left.51.1^{\circ} \mathrm{C}\right)$. We selected the most effective dose of opioid based on the Randall-Selitto test and performed the assay 30 and 60 minutes after opioid injection. The latency for the first lick or paw retraction of animals treated with morphine $(3 \mathrm{mg} / \mathrm{kg})$ was significantly increased at both temperatures after 30 minutes (Figure 6C) and 60 minutes (Supplemental Figure 14) when compared with untreated animals. Interestingly, treatment with hydrocodone $(10 \mathrm{mg} / \mathrm{kg})$ did not reduce the latency at $51.5^{\circ} \mathrm{C}$ and was not very effective when given 60 minutes before the test. As with mechanical nociception, coinjection with finasteride $(50 \mathrm{mg} / \mathrm{kg}$, i.p.) did not reduce the antinociceptive efficiency of either opioid at any temperatures or pretreatment times (Figure 6C and Supplemental Figure 14).

Finally, to test the effects of finasteride on the hyperalgesic effects of opioid withdrawal, we tested the effects of finasteride (50 $\mathrm{mg} / \mathrm{kg}$, i.p.) on the mechanical nociception induced by naloxone $(1.5 \mathrm{mg} / \mathrm{kg}$, i.p.) in rats subjected to SNL and treated with a 6-day escalating morphine treatment. Surprisingly, we found that finasteride significantly increased the force threshold for paw withdrawal only on the injured side (Figure 6D and Supplemental Figure 15), indicating that finasteride reduced the hyperalgesia associated with opioid withdrawal.

The fact that finasteride did not inhibit the antinociceptive or antiallodynic effect of either morphine or hydrocodone in response to different painful stimuli in a rat model of neuropathic pain demonstrates that finasteride is unlikely to interfere with the principal beneficial activity of opioids. Furthermore, the finding that finasteride reduced the hyperalgesia associated with opioid withdrawal strongly suggests it may be used to reduce the liability for opioid abuse and the untoward consequences of opioid withdrawal while retaining the clinical utility of opioids.

We also verified that finasteride $(50 \mathrm{mg} / \mathrm{kg}$, i.p.) did not alter the antinociceptive properties of morphine (3 $\mathrm{mg} / \mathrm{kg}$, s.c.) in non-lesioned rats, as assessed by the Randall-Selitto test (Supplemental Figure 16A) and the hot-plate test at $51.5^{\circ} \mathrm{C}$ (Supplemental Figure 16B). These data further suggest that finasteride did not diminish the antinociceptive properties of opioids.

Steroids regulate opioid self-administration. Because finasteride is a known $5 \alpha \mathrm{R}$ inhibitor, we hypothesized that finasteride reduc- 
A
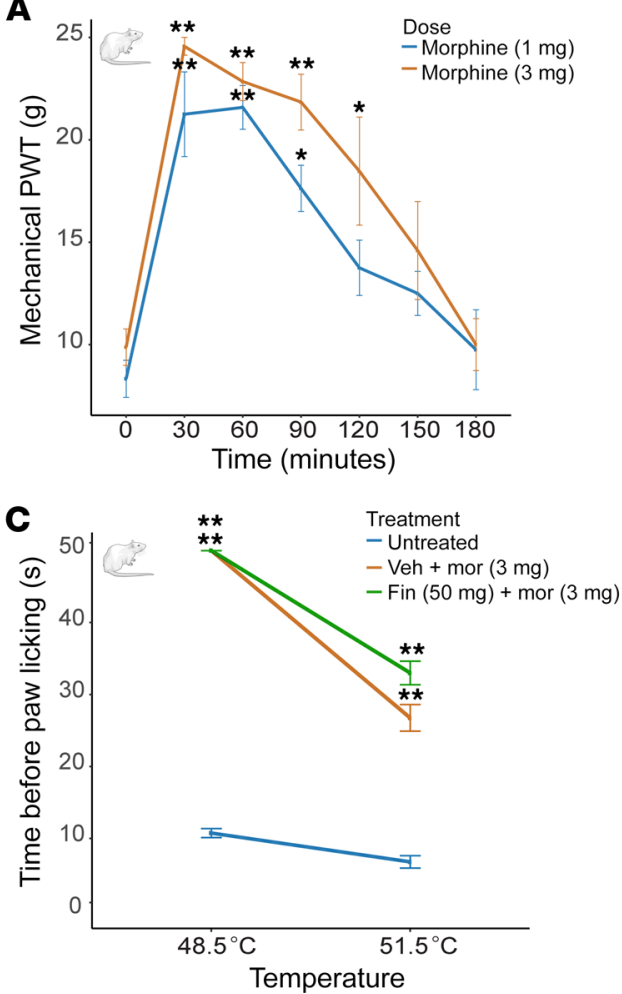
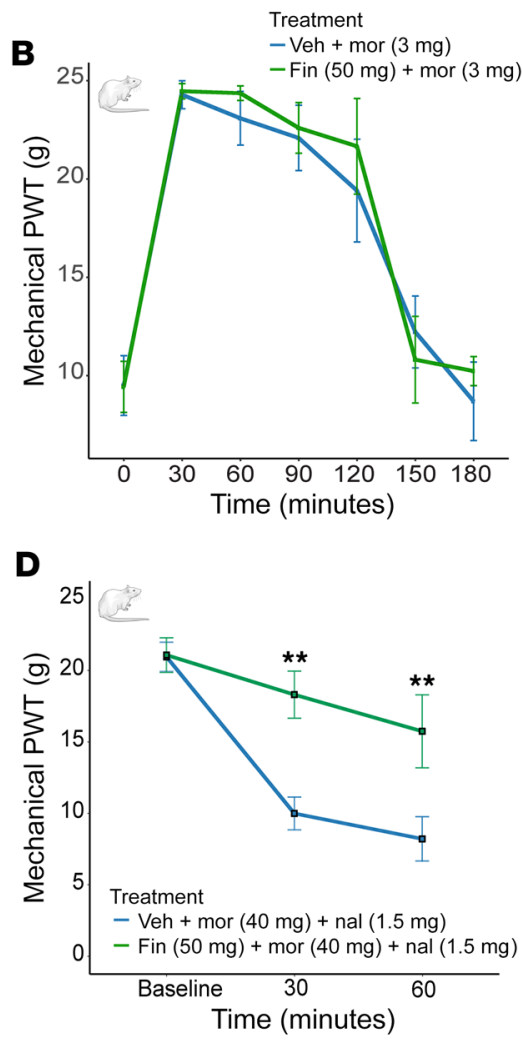

Figure 6. Finasteride does not affect the antinociceptive effect of opioids in a neuropathic pain model. (A) Paw-withdrawal thresholds (PWTs) in the Randall-Selitto test. Pain tolerance was measured over 180 minutes after treatment. Both doses of morphine significantly increased PWT compared with animals tested immediately before injection. Two-way ANOVA significant effect of time $[F(6,72)=31.09, P<$ $0.0001]$. Morphine $1 \mathrm{mg} / \mathrm{kg}: n=6$; morphine $3 \mathrm{mg} / \mathrm{kg}: n=8$ per condition. (B) Cotreatment with finasteride $(50 \mathrm{mg} / \mathrm{kg})$ did not block the antinociceptive effect of morphine $(3 \mathrm{mg} / \mathrm{kg})$. Two-way ANOVA significant effect of time $[F(6,48)=45.31, P<0.0001]$ but no significant effect of treatment $[F(1,8)=0.19, P=0.68]$ and no effect of interaction $[F(6,48)=0.37, P=0.89] . n=5$ rats per condition. (C) Finasteride did not affect the thermal antinociceptive effect of morphine as measured by the time before paw lick in response to different temperatures 30 minutes after treatment with morphine. Two-way ANOVA: at $48.5^{\circ} \mathrm{C}$, significant effect of treatment $[F(2,9)=770, P<0.0001]$; at $51.5^{\circ} \mathrm{C}$, significant effect of treatment $[F(2,9)=$ 33.12), $P<0.0001]$. The results for vehicle plus morphine and finasteride plus morphine were significantly different from those seen in the untreated animals. No difference was observed between vehicle plus morphine and finasteride plus morphine treatments. $n=4$ rats per condition. (D) Finasteride reduced hyperalgesia associated with opioid withdrawal. Rats subjected to SNL and a 6-day escalating morphine treatment were injected with finasteride $(50 \mathrm{mg} / \mathrm{kg}$, i.p.) or vehicle. Mechanical nociception was measured immediately on the injured paw after naloxone treatment and repeated 30 and 60 minutes later. Two-way ANOVA significant effect of interaction $[F(2,20)=4.53, P=0.024]$, time $[F(2,20)=19.4, P<0.0001]$, treatment $[\mathrm{F}(1,10)=13.1, P=0.0046] .{ }^{*} P<0.05,{ }^{* *} P<0.01$. Data are shown as the mean $\pm \mathrm{SEM}$. Adult male Sprague-Dawley rats were used to test the effect of finasteride treatment on morphine antinociceptive effects, and adult male Long-Evans rats were used for the naloxone-precipitated withdrawal experiment. These experiments were performed using a between-subject design.

es opioid self-administration by altering the level of one or more neuroactive steroids in the brain. To investigate the landscape of changes induced by the treatment of opioid-conditioned animals with finasteride and identify candidate neuroactive steroids regulating opioid intake, we isolated whole brains from treatment-naive and opioid-conditioned zebrafish treated with either DMSO or finasteride $(10 \mu \mathrm{M})$. We then performed steroid quantification using targeted ultra-performance liquid chromatography-mass spectrometry (UPLC-MS).

In other models, finasteride induces changes in steroid levels in specific brain regions $(45,46)$, but because of the small size of the zebrafish brain, we used whole brains and could not achieve the same level of regional specificity. Nonetheless, we identified interesting trends, including an accumulation of several $5 \alpha \mathrm{R}$ precursors and a reduction in $5 \alpha \mathrm{R}$ products after treatment with finasteride. We normalized the results (minimum-maximum) for each steroid and compared the levels between DMSOand finasteride-treated conditioned animals. The only steroid that reached significance by itself was DHEAS, which was markedly increased in finasteride-treated animals (Figure 7A). We also observed that other precursor steroids, including testosterone and pregnenolone, also showed the same trend of accumulating in finasteride-treated animals. Surprisingly, we also detected a reduction in dehydroepiandrosterone (DHEA), which could suggest an increase in the conversion of DHEA to DHEAS in treated animals. Interestingly, we did not detect the same trend for other sulfated steroid species (Supplemental Figure 17). The opposite trend of decreases in finasteride-treated animals was also observed for steroids downstream of $5 \alpha \mathrm{R}$, especially allopregnanolone and $3 \alpha$-androstanediol, but these differences did not reach statistical significance (Figure 7, $\mathrm{A}$ and $\mathrm{B})$.

We tested how the accumulation of DHEAS affects opioid self-administration. Given that sulfated steroids are less effective at crossing the bloodbrain barrier $(47,48)$, we incubated conditioned zebrafish with $10 \mu \mathrm{M}$ DHEA for 60 minutes before measuring opioid self-administration. As observed with finasteride, incubation with DHEA drastically reduced the number of visits at the active platform (Figure 8A). We also tested the primary DHEA precursor, pregnenolone, and again observed a reduction in opioid self-administration (Figure 8A). Taken together, these results suggest that the accumulation of DHEAS and other precursors observed in finasteride-treated animals could play an important role in the reduction of opioid self-administration. The fact that DHEA-treated animals had a reduced number of visits at the active platform also suggests that the reduction in DHEA level observed in finasteride-treated animals was a result of an increased conversion to DHEAS.

Since we detected a reduction in some products of $5 \alpha \mathrm{R}$ after treatment with finasteride, we decided to test whether their rein- 
A

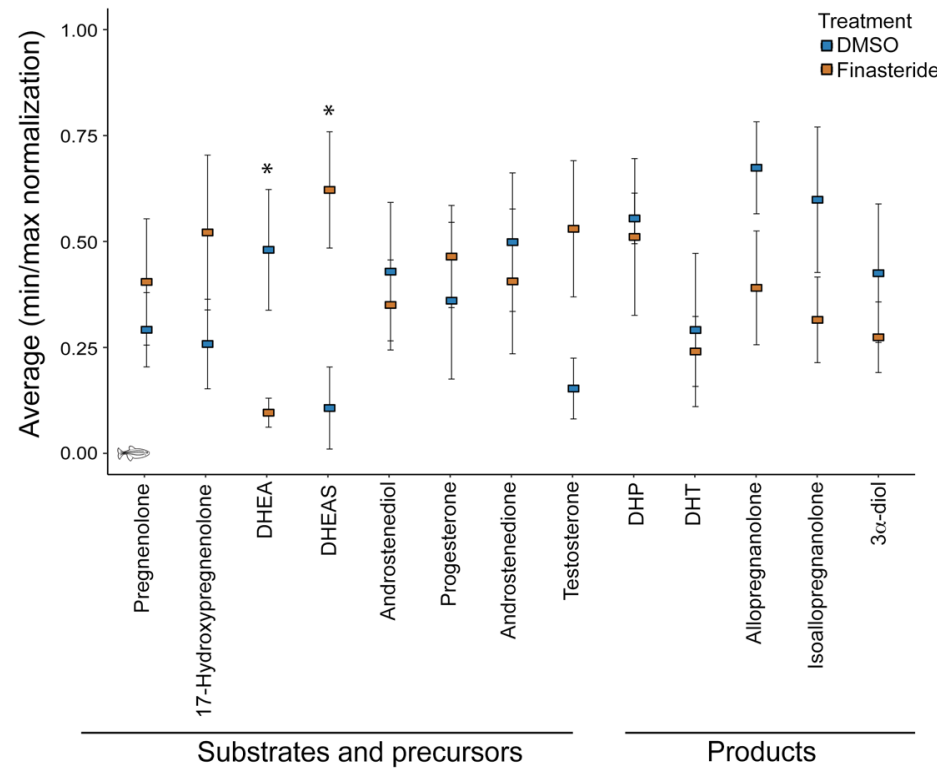

Figure 7. Finasteride treatment changes neurosteroid levels in the conditioned animal brain. (A) Normalization score for the quantification of steroids in conditioned brains treated with DMSO or finasteride $(10 \mu \mathrm{M}) . n=5$ set of 10 brains per condition. ${ }^{*} P<0.05$, by Student's $t$ test. (B) Partial neurosteroidogenesis pathways. Finasteride blocked the rate-limiting enzyme $5 \alpha$-reductase, causing accumulation of upstream neurosteroid species.

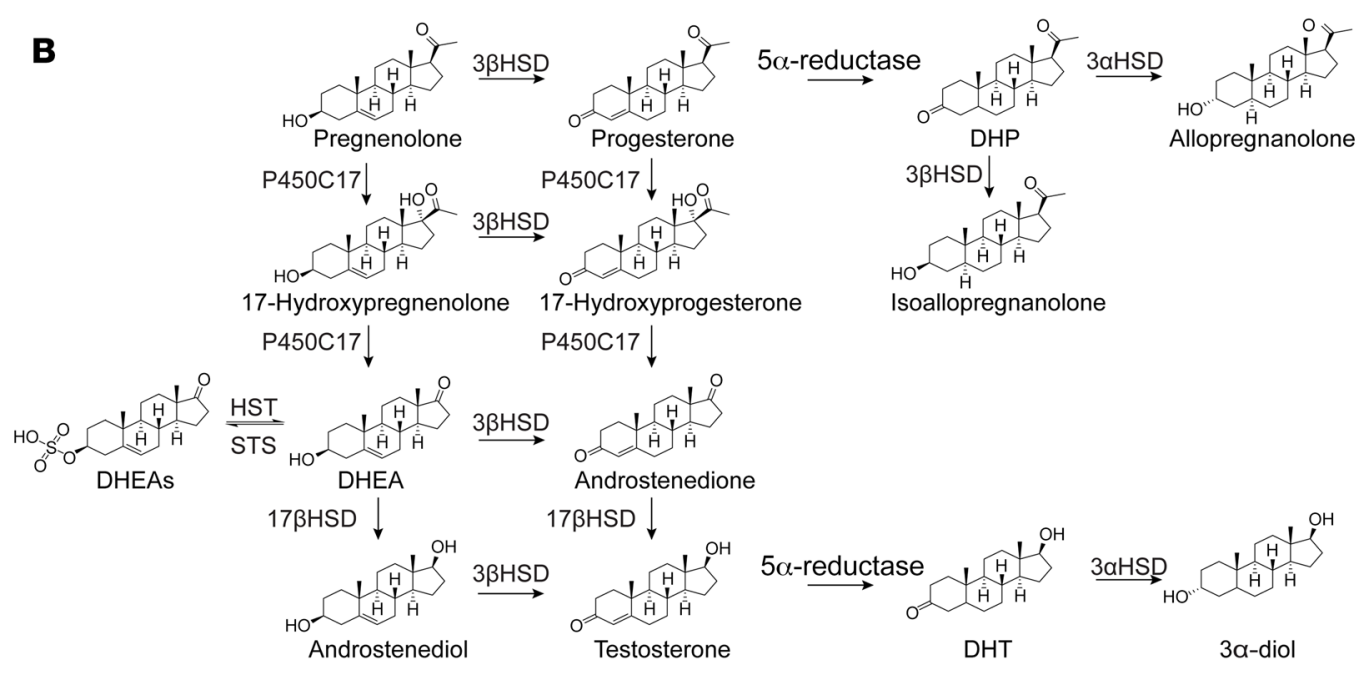

troduction could interfere with the activity of finasteride. However, since we did not detect any significant changes for a single product, we decided to test whether a combination of steroids from the same class could act together. We chose to cotreat conditioned animals with finasteride $(10 \mu \mathrm{M})$, allopregnanolone $(0.1 \mu \mathrm{M})$, androsterone $(1 \mu \mathrm{M})$, and $3 \alpha$-diol $(1 \mu \mathrm{M})$ for 60 minutes. Interestingly, the presence of these products was sufficient to partially block the effect of finasteride and significantly increase the number of visits at the active platform when compared with finasteride treatment alone (Figure 8B).

Taken together, these results suggest that the accumulation of DHEA or its sulfated form might play a critical role in mediating the effect of finasteride. However, other neurosteroids such as $5 \alpha \mathrm{R}$ products may also be involved in the regulation of opioid self-administration.

\section{Discussion}

We have demonstrated that finasteride modulated opioid consumption, a critical aspect of OUD, in 2 different animal models and for different classes of opioids. These results suggest that finasteride or finasteride-like molecules could be a viable therapeutic strategy to treat OUD. Although finasteride is typically used for treating nonneuronal indications, there is evidence in the literature to suggest it might also have beneficial effects in the nervous system. Rodents treated with finasteride exhibit reduced reactivity toward incentive stimuli and stress responses (49), both of which play important roles in substance abuse disorders $(50,51)$. Finasteride reduces risktaking behavior, a behavioral feature typically associated with substance use disorders $(49,52,53)$, and may help reduce pathological gambling (54). Since finasteride is FDA approved and its side effects and toxicology are well studied, it may be possible to rapidly initiate clinical trials to test the therapeutic potential of this drug for OUD. Furthermore, since finasteride did not reduce the antinociceptive effects of opioids and even opposed the hyperalgesia and physical signs associated with naloxone-precipitated opioid withdrawal, it could potentially be used as a treatment for OUD or an adjunct therapy for patients using opioids for pain relief.

We currently do not know whether finasteride influences opioid self-administration by reducing motivation for drug seeking or whether treated animals are simply satisfied with a lower amount of drug. 
A

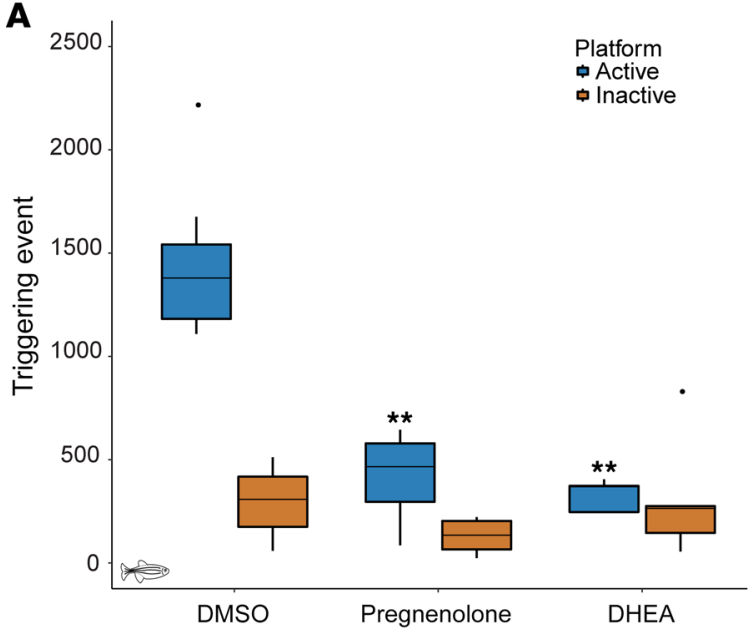

B

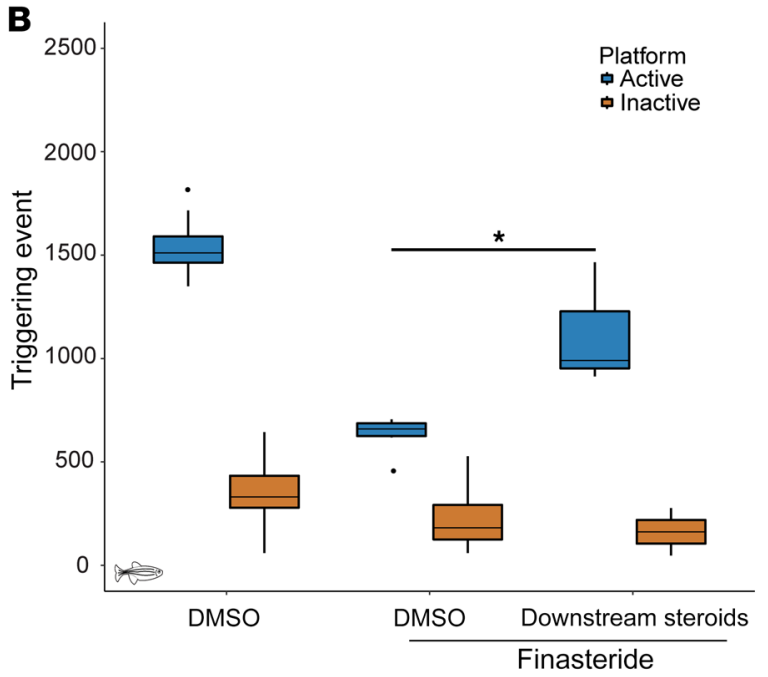

Figure 8. Specific neurosteroids also affect opioid self-administration. (A) Incubation with steroids upstream of $5 \alpha \mathrm{R}, \mathrm{DHEA}(10 \mu \mathrm{M})$ or pregnenolone (10 $\mu \mathrm{M})$, reduced the number of triggering events at the active platform. $P$ values were computed by Tukey's HSD test on 1-way ANOVA; inactive platform [F(2, $21)=2.18, P=0.14]$ and active platform $\left[F(2,21)=51.09, P=8.57 \times 10^{-9}\right]$. No significant difference was detected for the inactive platform compared with the DMSO control. DMSO: $n=12$, pregnenolone: $n=8$, and DHEA: $n=5$. (B) Cotreatment with finasteride $(10 \mu \mathrm{M})$ and a selection of steroids downstream of $5 \alpha$ R partially blocked the reduction in opioid self-administration induced by finasteride. $P$ values were computed by Tukey's HSD test on 1-way ANOVA; inactive platform $[F(2,16)=1.58, P=0.24]$ and active platform $\left[F(2,16)=68.93, P=1.37 \times 10^{-8}\right]$. No significant difference was detected for the inactive platform. DMSO: $n=8$, finasteride: $n=8$, finasteride plus downstream steroids: $n=3$. ${ }^{*} P<0.05,{ }^{*} P<0.01$. Each $n$ value represents a group of 15 animals. These experiments were performed using a between-subjects design. All box plots were generated using R graphic programming and the ggplot module. The lower and upper hinges correspond to the first and third quartiles. The line indicates the median. The whiskers extend from the hinges to the maximum or minimum value, at most 1.5 times the IQR from the hinge. Data points beyond that are considered outliers.

Our results in the fentanyl self-administration assay revealed that finasteride-treated rats initially performed nose-pokes to self-administer fentanyl at a rate similar to their untreated controls, but they slowed down their intake sooner than controls, suggesting finasteride-treated animals may achieve satiation with a lower amount of fentanyl.

Although the precise mechanism of finasteride action in opioid self-administration remains unknown, it is likely to involve changes in the steroid profile in the brain. Finasteride is an inhibitor of key enzymes in steroid production, the three $5 \alpha$-reductase isoenzymes SRD5A1, SRD5A2, and SRD5A3, which are expressed in different tissues, including the nervous system (55-59). Because the effects of finasteride on the steroid profile are pleiotropic (21, 22, 60 ), characterization of the specific steroid species involved in the regulation of opioid self-administration may eventually lead to the development of even more precise targeted therapies for OUDs.

Our results suggest that accumulation of DHEAS plays an important role in opioid self-administration. Finasteride treatment caused a significant decrease in the non-sulfated DHEA and an accumulation of DHEAS in the brain. Importantly, treatment of opioid-conditioned zebrafish with DHEA alone reduced opioid intake, further supporting the hypothesis that DHEA/ DHEAS plays an important role in opioid intake regulation. These neuroactive steroids have been shown to directly act on different neurotransmitter receptors; they can act as positive allosteric modulators of NMDA receptors, as negative modulators of the $\mathrm{GABA}_{\mathrm{A}}$ receptor, and as activators of other neuronal receptors such as $\sigma 1$ and TrkA (61-63). The modulation of these pathways has been shown to be important in the regulation of opioid abuse disorders $(18,64-69)$ and could explain why modulation of DHEA reduces opioid intake. Furthermore, previous reports showed that repeated DHEAS treatment prevents the development of opioid tolerance without showing an effect on self-administration (70). Additionally, chronic treatment with DHEA has also been shown to reduce cocaine self-administration and reinstatement in rats (71).

Although we believe that DHEA/DHEAS plays a key role in the effect of finasteride on opioid self-administration, we also have evidence that other steroid species could be involved. Similar to DHEA, these other steroids have been shown to affect key neuronal pathways relevant to substance abuse. For instance, the products of $5 \alpha \mathrm{R}, 3 \alpha$-diol and allopregnanolone, can act as positive allosteric modulators of GABA receptors (72-76) and have thus been linked to neuronal stress response $(77,78)$. Therefore, by reducing allopregnanolone production, finasteride may reduce the negative affective state that contributes to opioid self-administration.

In this study, we performed most of the rat experiments with males since finasteride is mainly prescribed in men (although there is some history of use to treat hirsutism in women, ref. 79), but our data suggest that both male and female rats showed reduced fentanyl self-administration upon finasteride treatment, raising the possibility that finasteride could be used as a treatment for OUD in both males and females, despite the differences in steroid levels between males and females (80).

Finasteride has been clinically used since the 1990s for the treatment of androgenic alopecia and benign prostate hyperplasia. Although it is considered a well tolerated and relatively safe drug, there is evidence of sexual disfunction in $3.4 \%$ to $15.8 \%$ 
of men. A rare but serious side effect known as post-finasteride syndrome (PFS) has also been reported (81-83). PFS prevalence is unclear but it manifests as a range of persistent physical and neuropsychiatric disorders such as depression and anxiety that develop during or after discontinuation of finasteride use. Clinical studies will be needed to fully elucidate the treatment regime and understand the side effects associated with the use of finasteride for the treatment of OUDs, and careful clinical consideration must be given in weighing potential risks and benefits of finasteride use.

The optimal human dose for OUD would also have to be determined. The finasteride doses we used in rats $(25-50 \mathrm{mg} / \mathrm{kg}$, i.p.) are considerably higher than the dose regimens of 1 to $5 \mathrm{mg} / \mathrm{d}$ used in humans for alopecia and benign prostatic hyperplasia, respectively. The rat doses were selected empirically based on previous studies conducted in the Bortolato laboratory showing that these doses are necessary to produce a significant reduction in $5 \alpha$ reduced metabolites in rodents after acute treatment (84). It has also been shown empirically that the doses used in this study produce ameliorative effects in rodent models of Tourette syndrome akin to those observed within a 3- to 6-week period of $5 \mathrm{mg} / \mathrm{d}$ dosage in patients with Tourette syndrome (84-86). It is important to note that the rat doses are single acute doses, whereas human dosing of finasteride is typically chronic. Finasteride is known to accumulate slowly with repeat dosing in humans, and the effects of finasteride persist for several days beyond what would be predicted based on compound half-life alone, which is attributed to persistent tight binding of finasteride to $5 \alpha \mathrm{R}$ (87). Taken together, these factors suggest that the human dose necessary to treat OUD would be much lower than the acute dose used in our rat studies, but the optimal dose would need to be established empirically.

In conclusion, the present study identified the widely used drug finasteride as an effective agent for reducing opioid intake in zebrafish and rat self-administration paradigms. The data further indicated that the DHEA/DHEAS pathway is a major mediator of finasteride's effect. These findings point to a promising potential therapeutic strategy in the fight against OUD and open new avenues for investigating the role of specific steroids in regulating opioid use behaviors.

\section{Methods}

\section{Zebrafish}

Adult fish treatment. Adult fish were transferred to a small treatment chamber (U.S. Plastic) with $100 \mathrm{~mL}$ of fish water, and the compound of interest was injected directly into the water. Fish were allowed to swim in the treatment solution for 1 hour prior to the self-administration assay.

Zebrafish self-administration. The same protocol as detailed in Bossé and Peterson (18) was used to condition fish in small groups of 15 animals. For the screen, fish were conditioned for 4 days and treated with the different compounds on day 5 before being tested in the arena for 30 minutes. For opioid conditioning, we used the following doses: hydrocodone and morphine $6 \mathrm{mg} / \mathrm{L}$ and fentanyl $0.12 \mathrm{mg} / \mathrm{L}$. Opioids were diluted in fish water. Between 60 and 100 animals were conditioned simultaneously and randomly assigned to different treatment conditions.

Food conditioning. The same apparatus was used as for hydrocodone conditioning. For food conditioning, fish were trained directly in the arena without performing the preconditioning protocol. Fish were trained for 50 minutes daily in a small group. Larvae food Ziegler 4 (VWR-Avantor) was suspended in fish water.

Steroid quantification. Conditioned fish were transferred to a small treatment chamber (U.S. Plastic) with $100 \mathrm{~mL}$ of fish water and treated with either DMSO $(0.02 \%)$ or finasteride $(10 \mu \mathrm{M})$. Fish were allowed to swim in the treatment solution for 1 hour. Treated animals were then transferred to a water bath with ice water for euthanasia. The brain of each animal was then extracted in PBS $1 \times$. The head was cut using a razor blade behind the gill, and the skull was then carefully peeled to expose the brain using surgical forceps. The brain was then extracted by performing a cut at the base of the cerebellum. The extracted tissue was then placed in $1.5 \mathrm{~mL}$ self-standing microcentrifuge tube (USA Scientific) on ice and the brains of 10 animals were pooled together in the same tube. Any liquid was then removed from each tube before weighing the tissues. Samples were then flash-frozen in liquid nitrogen and placed at $-80^{\circ} \mathrm{C}$ until extraction.

Reference standards were purchased from Steraloids. All solvents were HPLC grade, and all other chemicals used were of the highest grade available. Stock neurosteroid standard mixture was prepared by mixing $5 \mu \mathrm{L}$ of $1 \mathrm{mg} / \mathrm{mL}$ solution of each steroid and adjusting the final volume to $1 \mathrm{~mL}$ by using methanol (88-90). All the stock solutions were stored at $-80^{\circ} \mathrm{C}$.

Tissue samples were extracted as described previously (88-90). Briefly, tissue samples were extracted with $1 \mathrm{~mL}$ chloroform. The mixture was vortexed for 30 seconds and centrifuged for 5 minutes; the chloroform layer was transferred to a $2 \mathrm{~mL}$ tube and dried. The resulting residue was extracted with $1 \mathrm{~mL}$ methanol $(\mathrm{MeOH})$. The $\mathrm{MeOH}$ layer mixture after 5 minutes of centrifugation was added to the above chloroform extract. This mixture was dried and resuspended in $125 \mu \mathrm{L}$ $\mathrm{MeOH}$ and filtered using $5 \mathrm{kD}$ membrane filters. Filtrates were transferred to vials for UPLC-MS analysis.

Tissue sample extracts were subjected to UPLC-MS analysis for the measurement of neurosteroids as described previously (88-90). UPLC analyses were carried out using a Waters Acquity UPLC system connected with the high-performance triple quadrupole mass spectrometer. Analytical separations on the UPLC system were conducted using an Acquity UPLC C18 $1.6 \mu \mathrm{m}$ column $(2.1 \times 150 \mathrm{~mm})$ at a flow rate of $0.15 \mathrm{~mL} / \mathrm{min}$ and $\mathrm{C} 181.7 \mu \mathrm{m}$ column $(2.1 \times 50 \mathrm{~mm})$ at flow rate $0.2 \mathrm{~mL} / \mathrm{min}$. For the first column, the gradient was started with $100 \% \mathrm{~A}\left(0.1 \%\right.$ formic acid in $\left.\mathrm{H}_{2} \mathrm{O}\right)$ and $0 \% \mathrm{~B}(0.1 \%$ formic acid in $\mathrm{CH}_{3} \mathrm{CN}$ ), after 0.1 minute changed to $80 \%$ A over 1 minute, and then $45 \%$ A over 5 minutes, followed by $20 \%$ A in 2 minutes. Finally, over 0.5 minutes, it was changed to $0 \% \mathrm{~A}$, then after 13 minutes, it was changed to the original $100 \%$ A over 1 minute, resulting in a total separation time of 13 minutes. For the second column, the gradient was started with $100 \% \mathrm{~A}\left(0.1 \%\right.$ formic acid in $\left.\mathrm{H}_{2} \mathrm{O}\right)$ and $0 \% \mathrm{~B}$ (0.1\% formic acid in $\mathrm{CH}_{3} \mathrm{OH}$ ), after 0.1 minute changed to $80 \% \mathrm{~A}$ over 2 minutes, and then $45 \%$ A over 2 minutes, followed by $20 \% \mathrm{~A}$ in 2 minutes. Finally, over 1 minute, it was changed to $0 \% \mathrm{~A}$, and then after 8 minutes, it was changed to the original $100 \%$ A over 2 minutes, resulting in a total separation time of 10 minutes. The elution from the UPLC column was introduced to the mass spectrometer. All MS experiments were performed by using electrospray ionization (ESI) in both positive ion and negative ion mode, with an ESI-MS capillary voltage of $3.5 \mathrm{kV}$, an extractor cone voltage of $3 \mathrm{~V}$, and a detector voltage of $650 \mathrm{~V}$. The following MS conditions were used: desolvation gas at $400 \mathrm{~L} / \mathrm{h}$, desolvation temperature at $350^{\circ} \mathrm{C}$, and source tempera- 
ture $150^{\circ} \mathrm{C}$. Pure standards of all targeted neurosteroids were used to optimize the UPLC-MS/MS conditions prior to performing analysis and calibration curves (88-90). Reference standards were run before the first sample, in the middle of the runs, and after the last sample to prevent errors due to matrix effect and day-to-day instrument variations. In addition, spiked samples were also run before the first sample and after the last sample to calibrate for the drift in the retention time of all neurosteroids due to the matrix effect. After standard and spiked sample runs, several blanks were injected to wash the injector and avoid carryover effects. Resulting data were processed by using Target Lynx 4.1 software (Waters) (88-90).

Steroid counts were first normalized using the initial weight of the tissue before extraction. To compare the levels of the steroids, we then used minimum-maximum normalization for steroid count in each sample.

\section{Rats}

Hydrocodone self-administration and nociception. Hydrocodone (Spectrum Chemical), morphine (Spectrum Chemical), and naloxone (Tocris, Bio-Techne) were dissolved in a solution of $2 \%$ DMSO and $98 \%$ saline. Finasteride (Astatech) was suspended in a solution of $5 \%$ DMSO, $5 \%$ Tween 80, and 90\% saline (5:5:90).

The self-administration apparatus consisted of 8 operant conditioning chambers (Habitest, Coulbourn), measuring $30.48 \mathrm{~cm}(\mathrm{~W}) \times$ $25.4 \mathrm{~cm}(\mathrm{D}) \times 30.48 \mathrm{~cm}(\mathrm{H})$ and enclosed in sound-attenuating cubicles with ventilation fans. Each chamber was equipped with 2 retractable levers: an active lever coupled to the i.v. delivery of hydrocodone and a control (inactive) lever. Active lever placement on the left or right side followed a counterbalanced order. Three cue lights were placed over the active lever. The apparatus was controlled by Graphic State 4 software.

The experimental procedure was as follows: Opioid self-administration was performed using a modified version of the protocol described by Mavrikaki et al. (91). Rats weighing 225 to $250 \mathrm{~g}$ were used. Sprague-Dawley male rats (Charles River Laboratories) were anesthetized with ketamine and xylazine and underwent catheterization surgery. Briefly, a polyurethane catheter was inserted through the external jugular vein, passed under the skin, and fixed in the midscapular region. Postoperative care included buprenorphine and enrofloxacin for analgesic and antibiotic management, respectively. Catheter patency was maintained through daily flushing with a heparin (500 $\mathrm{IU} / \mathrm{mL}$ ) $/ 50 \%$ dextrose solution.

Ten days after surgery, all rats were gently handled and kept under a food restriction regimen that maintained them at $90 \%$ of their initial body weight and was continued throughout the whole behavioral procedure. A syringe containing a hydrocodone solution was placed in an infusion pump located outside the chamber and connected to the rat's catheter via a fluid swivel and spring-covered Tygon tube suspended through a counterbalanced swivel. The solution was administered at a dose of 0.016 to $0.128 \mathrm{mg} / \mathrm{kg} /$ infusion in a volume of $160 \mu \mathrm{L} / \mathrm{kg} /$ infusion. Operant training began 3 days later and consisted of 3 stages of an FR reinforcement schedule: FR1, FR2, and FR5. Rats underwent daily 1-hour long experimental sessions between 9:00 am and 3:00 pm and for 7 days per week consisting of a sequence of trials (Figure 3). Each trial began with a 5-second period, during which the house light was turned off and the cue light blinked 3 consecutive times. Subsequently, the house light was turned on and both levers were extended. After the rat completed the FR on either lever, both levers retracted, and a new trial began after a 15-second time-out period. Each rat progressed from FR1 to FR2 and from FR2 to FR5 after reaching stability, defined as more than $70 \%$ of total lever presses on the active lever for 3 consecutive days. FR1 and FR2 stability criteria were reached from day 12 through 19 and from day 15 through day 23 of training, respectively. All animals reached FR5 stability by day 31 of training and were treated with either finasteride or its vehicle.

Naloxone-precipitated opioid withdrawal. Long Evans male rats (180-225 g) (Charles River Laboratories) received s.c. injections of morphine with the regimen previously described (cumulative doses of $5,10,20,30$, and $40 \mathrm{mg} / \mathrm{kg}$ per day within 5 days). On day 6 , rats received an acute dose of morphine $(40 \mathrm{mg} / \mathrm{kg}$, s.c.), followed by either finasteride $(50 \mathrm{mg} / \mathrm{kg}$, i.p., 100 minutes later) or its vehicle and naloxone $(1.5 \mathrm{mg} / \mathrm{kg}$, i.p., 120 minutes after the first injection). Rats were immediately placed inside a Plexiglass chamber with bedding; animals were video-recorded for 30 minutes, and blinded observers monitored their opioid withdrawal signs, including wet-dog shakes, jumps, grooming, and digging.

Neuropathic pain assessment. For SNL, Sprague-Dawley rats (Charles River Laboratories) weighing 150 to $180 \mathrm{~g}$ were used. After 2 to 3 days of handling, rats underwent mechanical nociception testing via von Frey hair and Randall-Selitto tests. Neuropathy was then induced by SNL surgery as previously described (41). Rats were anesthetized using xylazine and ketamine $(10 / 75 \mathrm{mg} / \mathrm{kg}$, i.p.), and their left L5 spinal nerve was exposed and tightly ligated with 4.0 silk suture (Mersilk, Ethicon thread, Johnson \& Johnson). Muscle, fascia, and skin were then sutured, and the rats were treated with enrofloxacin $(10 \mathrm{mg} / \mathrm{kg}$, s.c.) and carprofen $(5 \mathrm{mg} / \mathrm{kg}$, s.c.) for postoperative care. Fourteen days after surgery, nociception was retested, and allodynia was confirmed in rats exhibiting a greater than $30 \%$ reduction of their pain threshold. Rats were then assigned to different treatment groups to receive either morphine (1-3 mg/ $\mathrm{kg}$, s.c.), hydrocodone (1-10 mg/ $\mathrm{kg}$, s.c.), or saline. The antinociceptive effects of opioids were tested every 30 minutes for 6 consecutive observations. The analgesic effects of morphine and hydrocodone (at their most effective doses) were also tested in combination with finasteride $(50 \mathrm{mg} / \mathrm{kg}$, i.p.) or its vehicle to ascertain whether finasteride altered the antiallodynic properties of opioids. The effects of opioids and finasteride were also tested for thermal nociception in a separate group of rats with SNL using the hot-plate procedure.

Tactile allodynia was assessed using a set of 8 von Frey monofilaments (Bioseb) with logarithmic incremental stiffness (of 1.4, 2, 4, $6,8,10,15$, and $26 \mathrm{~g}$ ). Paw-withdrawal threshold was measured, and $50 \%$ response threshold was calculated using the Up-Down method and Dixon's formulae as previously described (42). Behavioral assessments were run prior to and 14 days after SNL surgery. Rats were individually placed in Plexiglass compartments $(17 \times 11 \times 13 \mathrm{~cm})$ with a wire mesh bottom that allowed full access to paws. After 20-30 minutes of acclimation, the first $6 \mathrm{~g}$ hair was perpendicularly applied against the plantar surface of the left hind paw for 6 seconds. Paw withdrawal and/or licking reflex was considered as a positive response. Depending on the positive or negative response, the next filament with either lower or higher force was tested, respectively. Testing continued until either 4 consecutive negative or 5 consecutive positive responses were recorded after the first change of direction. 
Nociceptive withdrawal threshold was assessed using the RandallSelitto algesimeter (Ugo Basile) as previously described (43). After daily handling and acclimation to the apparatus, rats were wrapped in a cotton cloth and immobilized. The medial portion of the plantar surface of the left hind paw was carefully placed on the device's tip. An increasing mechanical force was applied until a withdrawal response was observed. The paw-withdrawal threshold for the Randall-Selitto experiment was set at $25 \mathrm{~g}$ of force applied. Rats were tested every 30 minutes for 3 consecutive hours after treatment (6 applications in total). For the assessment of morphine withdrawal-induced hyperalgesia, rats with SNL were subjected to a cumulative morphine treatment (as described above) for 5 days. On day 6, the effects of finasteride on naloxone-precipitated opioid withdrawal were tested using the Randall-Selitto algesimeter 30 minutes after morphine injection, as well as 30 and 60 minutes later. Testing was performed in lesioned and non-lesioned rats.

Thermal nociception was assessed using the hot-plate analgesia meter (IITC Life Science). The rat was placed on a plate maintained at different temperatures $\left(48.5^{\circ} \mathrm{C}\right.$ and $\left.51.5^{\circ} \mathrm{C}\right)$, and their progressive latencies to lick the left hind paw were measured. Testing was performed in both lesioned and non-lesioned rats.

Fentanyl self-administration. A total of 20 adult male and adult female Wistar rats (Charles River Laboratories) weighing 200-475 g at the start of the experiment were individually housed and kept on a 12-hour light/ 12-hour dark cycle in a temperature- and humiditycontrolled room. Animals were provided food and water ad libitum.

Fentanyl citrate (Medisca) was dissolved in deionized water at a concentration of $50 \mu \mathrm{g} / \mathrm{mL}$. Finasteride (Astatech) was suspended (50 $\mathrm{mg} / \mathrm{mL}$ ) in a solution of $2.5 \%$ ethanol, $5 \%$ Tween 80 , and $92.5 \%$ saline.

Oral fentanyl self-administration tasks were completed in 8 modular operant chambers (Med Associates) equipped with a liquid magazine tray stationed between 2 nose-poke devices. Additionally, the operant chamber was outfitted with a solenoid-controlled liquid valve (Lee Valves) and a set of audiovisual cue equipment (house light, magazine light, and a tone generator).

The experimental procedure was as follows: An operant oral self-administration behavioral assay described by Shaham et al. (92) was utilized. Rats were trained to obtain liquid fentanyl delivered into a liquid magazine tray after an operant response on an FR1 reinforcement schedule. During the self-administration sessions, a nose-poke in the active port (counterbalanced between animals) resulted in fentanyl delivery $(0.02 \mathrm{mg} / \mathrm{kg} /$ delivery $)$. Concurrent with drug delivery was a 10-second audiovisual conditioned stimulus (CS) consisting of a 1-second illumination of a light inside the nose-poke port, a 10-second tone, and a 10-second illumination of a light stationed above the liquid magazine tray. Any additional nose-pokes during the 10-second CS were without consequence. Drug availability at the start of each session and after CS presentations was signaled by illumination of the house-light placed on the wall opposite of the nose-poke ports. All nose-pokes in the inactive port were without consequence. Animals were given two 30-minute magazine training sessions, during which any active responses resulted in fentanyl and CS presentation; however, if the animal made no responses within 2-3 minutes of the last drug delivery (or start of the session) a noncontingent fentanyl and CS delivery occurred. After these 2 training sessions, animals had fifteen 1-hour sessions to self-administer fentanyl for 5 days per week. During these 15 sessions, all animals reached a response criterion of more than $70 \%$ of nose-pokes occurring at the active nose-poke port. To test the effect of finasteride on fentanyl consumption, animals received 10 additional self-administration sessions across 12 days, in which finasteride $(50 \mathrm{mg} / \mathrm{kg}, 1 \mathrm{~mL} / \mathrm{kg})$ or vehicle $(1 \mathrm{~mL} / \mathrm{kg})$ was i.p. administered 45 minutes prior to the session. Each treatment (vehicle or finasteride) was given for 5 consecutive days, with a 48 -hour period before the animal received the opposite treatment. The order of treatment administration was counterbalanced across animals. After self-administration sessions, fentanyl consumed $(\mathrm{mg} / \mathrm{kg})$ was calculated for each animal by subtracting the amount of fentanyl left in the magazine tray from the total amount of drug delivered.

Statistics. For zebrafish self-administration data, R graphic programming was used to generate the plots. ANOVA tests were run on plot data to test significance. ANOVA tests were performed first on inactive platforms for each data set to validate that there was no difference between the different conditions, and then active platform values were used to test for significance. All box plots were generated using R graphic programming and the ggplot module. The lower and upper hinges correspond to the first and third quartiles. The line is the median. The whiskers extend from the hinges to the maximum or minimum value at most 1.5 times the $\mathrm{IQR}$ from the hinge. Data points beyond that are considered outliers. No data points were excluded from the statistical analysis. For the experiment in rats, the specific statistical test has been specified in each figure legend along with what the error bar represented for each figure. A $P$ value of less than 0.05 was considered significant.

Study approval. All animal studies were approved by the University of Utah and the University of Washington IACUCs. All zebrafish experiments were approved by the University of Utah IACUC. Hydrocodone self-administration studies and nociception studies in rats were compliant with NIH guidelines and approved by the IACUC of the University of Utah. The fentanyl self-administration studies in rats were conducted under the guidance and approval of the IACUC at the University of Washington and pursuant to federal regulations regarding work with animals.

\section{Author contributions}

GDB designed the experiments and performed the zebrafish assays, analyzed the data, and wrote the manuscript with RTP and MB. $\mathrm{RC}$ designed the nociceptive experiments in rats and performed the experiments. GF designed and performed the hydrocodone self-administration assay in rats assisted by EV. TZ contributed to experiment design. RTP designed and supervised zebrafish experiments. MB designed, analyzed, and supervised the experiments on rat hydrocodone self-administration experiments and nociception. NWG performed steroid extraction and quantification. RDF and JSL designed and performed the fentanyl self-administration experiment in rats. RDF and PEMP designed and analyzed the fentanyl self-administration in rats. All authors contributed to data interpretation and commented on the manuscript.

\section{Acknowledgments}

We thank the National Institute on Drug Abuse (NIDA) Drug Supply Program for providing hydrocodone bitartrate powder. We thank Chelsea Herdman for helpful comments and bioinformatic support. We thank Karen Odeh and Stefanos Loizou for their technical support. We would like to acknowledge the Centralized 
Zebrafish Animal Resource (CZAR) at the University of Utah for providing zebrafish husbandry, laboratory space, and equipment to carry out portions of this research. Expansion of the CZAR is supported in part by NIH grant 1G20OD018369-01. This work was supported by the Charles and Ann Sanders Research Scholar Award, the L.S. Skaggs Presidential Endowed Chair, and NIH grant R21DA049530 (to MB and RTP). Oral rodent fentanyl studies were supported by NIDA R01-DA039687. GDB was supported by the Canadian Institutes of Health Research (CIHR) fellowship program. RDF was supported by NIDA (T32-DA007278). TZ was supported by the National Human Genome Research Institute
(T32 HG008962). Research reported in this publication was supported by the National Center for Advancing Translational Sciences of the NIH under award number UL1TR002538. The content is solely the responsibility of the authors and does not necessarily represent the official views of the NIH.

Address correspondence to: Randall T. Peterson or Marco Bortolato, University of Utah, College of Pharmacy, 30 South 2000 East, Salt Lake City, Utah 84112, USA. Phone: 801.581.3402; Email: randall.peterson@pharm.utah.edu (RTP). Phone: 801.587.3352; Email:marco.bortolato@utah.edu (MB).
1. Wilson N, et al. Drug and opioid-involved overdose deaths - United States, 2017-2018. MMWR Morb Mortal Wkly Rep. 2020;69(11):290-297.

2. Scholl L, et al. Drug and opioid-involved overdose deaths - United States, 2013-2017. MMWR Morb Mortal Wkly Rep. 2018;67(5152):2013-2017.

3. Mattick R, et al. Buprenorphine maintenance versus placebo or methadone maintenance for opioid dependence. Cochrane Database Syst Rev. 2004;(3):CD002207.

4. Brady KT, et al. Prescription opioid misuse, abuse, and treatment in the united states: an update. Am J Psychiatry. 2016;173(1):18-26.

5. Zhou Z, et al. Gene expression in the addicted brain. Int Rev Neurobiol. 2014;116:251-273.

6. Feltenstein MW, et al. Neural substrates and circuits of drug addiction. Cold Spring Harb Perspect Med. 2021;11(4):a039628.

7. Stewart AM, et al. Developing zebrafish models of autism spectrum disorder (ASD). Prog Neuropsychopharmacol Biol Psychiatry. 2014;50:27-36.

8. Shams $\mathrm{S}$, et al. The zebrafish as a promising tool for modeling human brain disorders. A review based upon an IBNS Symposium. Neurosci Biobehav Rev. 2018;85:176-190.

9. Levin ED, et al. Perspectives on zebrafish neurobehavioral pharmacology. Pharmacol Biochem Behav. 2015;139:93.

10. Darland T, Dowling JE. Behavioral screening for cocaine sensitivity in mutagenized zebrafish. Proc Natl Acad Sci USA. 2001;98(20):11691-11696.

11. Lau B, et al. Dissociation of food and opiate preference by a genetic mutation in zebrafish. Genes Brain Behav. 2006;5(7):497-505.

12. Kedikian X, et al. Behavioral and molecular analysis of nicotine-conditioned place preference in zebrafish. PLoS One. 2013;8(7):e69453.

13. Faillace MP, et al. Evaluation of the rewarding properties of nicotine and caffeine by implementation of a five-choice conditioned place preference task in zebrafish. Prog Neuro-Psychopharmacology Biol Psychiatry. 2018;84(pt A):160-172.

14. Cousin MA, et al. Larval zebrafish model for FDA-approved drug repositioning for tobacco dependence treatment. PLoS One. 2014;9(3):e90467.

15. MacRae CA, Peterson RT. Zebrafish-based small molecule discovery. Chem Biol. 2003;10(10):901-908.

16. Li Y, et al. Zebrafish: a promising in vivo model for assessing the delivery of natural products, fluorescence dyes and drugs across the blood-brain barrier. Pharmacol Res. 2017;125(Pt B):246-257.
17. Stewart AM, et al. Molecular psychiatry of zebrafish. Mol Psychiatry. 2015;20(1):2-17.

18. Bossé GD, Peterson RT. Development of an opioid self-administration assay to study drug seeking in zebrafish. Behav Brain Res. 2017;335:158-166.

19. Aggarwal S, et al. An overview on 5alpha-reductase inhibitors. Steroids. 2010;75(2):109-153.

20. Faller B, et al. Finasteride: a slow-binding $5 \alpha$-reductase inhibitor. Biochemistry. 1993;32(21):5705-5710.

21. Uygur MC, et al. Effects of the $5 \alpha$-reductase inhibitor finasteride on serum levels of gonadal, adrenal, and hypophyseal hormones and its clinical significance: a prospective clinical study. Steroids. 1998;63(4):208-213.

22. Drake $\mathrm{L}$, et al. The effects of finasteride on scalp skin and serum androgen levels in men with androgenetic alopecia. JAm Acad Dermatol. 1999;41(4):550-554.

23. Dusková M, et al. Finasteride treatment and neuroactive steroid formation. Prague Med Rep. 2009;110(3):222-230.

24. Martini L, et al. Androgen and progesterone metabolism in the central and peripheral nervous system. J Steroid Biochem Mol Biol. 1993;47(1-6):195-205.

25. Kim EH, et al. ScienceDirect the use of 5 -alpha reductase inhibitors in the treatment of benign prostatic hyperplasia. Asian JUrol. 2018;5(1):28-32

26. Roehrborn CG, et al. Efficacy and safety of a dual inhibitor of 5 -alpha-reductase types 1 and 2 (dutasteride) in men with benign prostatic hyperplasia. Urology. 2002;60(3):434-441.

27. Diotel N, et al. Activity and expression of steroidogenic enzymes in the brain of adult zebrafish. Eur J Neurosci. 2011;34(1):45-56.

28. Diotel N, et al. Aromatase in the brain of teleost fish: expression, regulation and putative functions. Front Neuroendocrinol. 2010;31(2):172-192.

29. Weger M, et al. Expression and activity profiling of the steroidogenic enzymes of glucocorticoid biosynthesis and the fdx 1 co-factors in zebrafish. J Neuroendocrinol. 2018;30(4):1-15

30. Zhang Q, et al. Zebrafish cyp11c1 knockout reveals the roles of 11-ketotestosterone and cortisol in sexual development and reproduction. Endocrinology. 2020;161(6):bqaa048.

31. Sakamoto $\mathrm{H}$, et al. Activity and localization of $3 \beta$-hydroxysteroid dehydrogenase $/ \delta 5-\delta 4$-isomerase in the zebrafish central nervous system. J Comp Neurol. 2001;439(3):291-305.

32. Pasmanik M, Callard GV. Aromatase and $5 \alpha$-reductase in the teleost brain, spinal cord, and pituitary gland. Gen Comp Endocrinol.
1985;60(2):244-251.

33. Diotel N, et al. The brain of teleost fish, a source, and a target of sexual steroids. Front Neurosci. 2011;5:137.

34. Raj B, et al. Simultaneous single-cell profiling of lineages and cell types in the vertebrate brain. Nat Biotechnol. 2018;36(5):442-450.

35. Simpson EH, Balsam PD. The behavioral neuroscience of motivation: an overview of concepts, measures, and translational applications. Brain Imaging Behav Neurosci. 2015;(27):1-12.

36. Ko MC, et al. Relative reinforcing effects of three opioids with different durations of action. J Pharmacol Exp Ther. 2002;301(2):698-704.

37. Volpe DA, et al. Uniform assessment and ranking of opioid Mu receptor binding constants for selected opioid drugs. Regul Toxicol Pharmacol. 2011;59(3):385-390.

38. Suzuki J, El-Haddad S. A review: fentanyl and non-pharmaceutical fentanyls. Drug Alcohol Depend. 2017;171:107-116.

39. Budziszewska B, et al. Neurosteroids and the naloxone-precipitated withdrawal syndrome in morphine-dependent mice. Eur Neuropsychopharmacol.1996;6(2):135-140.

40. Verdi J, Ahmadiani A. Finasteride, a $5 \alpha$-reductase inhibitor, potentiates antinociceptive effects of morphine, prevents the development of morphine tolerance and attenuates abstinence behavior in the rat. Horm Behav. 2007;51(5):605-610.

41. Ho Kim S, Mo Chung J. An experimental model for peripheral neuropathy produced by segmental spinal nerve ligation in the rat. Pain. 1992;50(3):355-363.

42. Chaplan SR, et al. Quantitative assessment of tactile allodynia in the rat paw. J Neurosci Methods. 1994;53(1):55-63.

43. Santos-Nogueira E, et al. Randall-Selitto test: new approach for the detection of neuropathic pain after spinal cord injury. J Neurotrauma. 2012;29(5):898-904.

44. Rácz I, Zimmer A. Animal models of nociception. In: Hrabe de Angelis M, et al., eds. Standards of Mouse Model Phenotyping. Wiley-VCH Verlag $\mathrm{GmbH} ; 2006: 221-235$.

45. Soggiu A, et al. Exploring the neural mechanisms of finasteride: a proteomic analysis in the nucleus accumbens. Psychoneuroendocrinology. 2016;74:387-396.

46. Giatti S, et al. Effects of subchronic finasteride treatment and withdrawal on neuroactive steroid levels and their receptors in the male rat brain Neuroendocrinology. 2016;103(6):746-757. 
47. Qaiser MZ, et al. Uptake and metabolism of sulphated steroids by the blood-brain barrier in the adult male rat. J Neurochem. 2017;142(5):672-685.

48. Kishimoto Y, Hoshi M. Dehydroepiandrosterone sulphate in rat brain: incorporation from blood and metabolism in vivo. J Neurochem. 1972;19(9):2207-2215

49. Godar SC, et al. The steroidogenesis inhibitor finasteride reduces the response to both stressful and rewarding stimuli. Biomolecules. 2019;9(11):1-16.

50. Hollon NG, et al. Stress effects on the neural substrates of motivated behavior. Nat Neurosci. 2015;18(10):1405-1412.

51. Zorrilla EP, et al. Corticotropin releasing factor: a key role in the neurobiology of addiction. Front Neuroendocrinol. 2014;35(2):234-244.

52. MacPherson L, et al. Changes in sensation seeking and risk-taking propensity predict increases in alcohol use among early adolescents. Alcohol Clin Exp Res. 2010;34(8):1400-1408.

53. Sher KJ, et al. Personality and substance use disorders: a prospective study. JConsult Clin Psychol. 2000;68(5):818-829.

54. Bortolato M, et al. Finasteride attenuates pathological gambling in patients with Parkinson disease. JClin Psychopharmacol. 2012;32(3):424-425.

55. Normington K, Russell DW. Tissue distribution and kinetic characteristics of rat steroid $5 \alpha$-reductase isozymes. Evidence for distinct physiological functions. J Biol Chem. 1992;267(27):19548-19554.

56. Celotti F, et al. The $5 \alpha$-reductase in the brain: molecular aspects and relation to brain function. Front Neuroendocrinol.1992;13(2):163-215.

57. Thigpen AE, et al. Tissue distribution and ontogeny of steroid $5 \alpha$-reductase isozyme expression. J Clin Invest. 1993;92(2):903-910.

58. Agís-Balboa RC, et al. Characterization of brain neurons that express enzymes mediating neurosteroid biosynthesis. Proc Natl Acad Sci U S A. 2006;103(39):14602-14607.

59. Yamana $\mathrm{K}$, et al. Human type $35 \alpha$-reductase is expressed in peripheral tissues at higher levels than types 1 and 2 and its activity is potently inhibited by finasteride and dutasteride. Horm Mol Biol Clin Investig. 2010;2(3):293-299.

60. Mukai Y, et al. Studies on neurosteroids XXV. Influence of a 5alpha-reductase inhibitor, finasteride, on rat brain neurosteroid levels and metabolism. Biol Pharm Bull. 2008;31(9):1646-1650.

61. Prough RA, et al. Novel mechanisms for DHEA action. JMol Endocrinol. 2016;56(3):R139-R155.

62. Dong Y, Zheng P. Dehydroepiandrosterone sulphate: action and mechanism in the brain.
J Neuroendocrinol. 2012;24(1):215-224.

63. Maninger $\mathrm{N}$, et al. Neurobiological and neuropsychiatric effects of DHEA and DHEA-S. Front Neuroendocrinol. 2009;30(1):65-91.

64. Ramshini E, et al. The role of GABAB receptors in morphine self-administration. Int J Prev Med. 2013;4(2):158-164.

65. LaLumiere RT, Kalivas PW. Glutamate release in the nucleus accumbens core is necessary for heroin seeking. J Neurosci. 2008;28(12):3170-3177.

66. Baik JH. Dopamine signaling in reward-related behaviors. Front Neural Circuits. 2013;7:1-16.

67. Xi ZX, Stein EA. Increased mesolimbic GABA concentration blocks heroin self-administration in the rat. J Pharmacol Exp Ther. 2000;294(2):613-619.

68. You ZB, et al. Dopamine D3R antagonist VK4-116 attenuates oxycodone self-administration and reinstatement without compromising its antinociceptive effects. Neuropsychopharmacology. 2019;44(8):1415-1424.

69. Koob GF. Neurobiology of opioid addiction: opponent process, hyperkatifeia, and negative reinforcement. Biol Psychiatry. 2020;87(1):44-53.

70. Ren X, et al. A neuroactive steroid, dehydroepiandrosterone sulfate, prevents the development of morphine dependence and tolerance via c-fos expression linked to the extracellular signal-regulated protein kinase. Behav Brain Res. 2004;152(2):243-250.

71. Maayan R, et al. Dehydroepiandrosterone attenuates cocaine-seeking behaviour independently of corticosterone fluctuations. J Neuroendocrinol. 2015;27(11):819-826.

72. Tomaselli G, Vallée M. Stress and drug abuserelated disorders: the promising therapeutic value of neurosteroids focus on pregnenolone-progesterone-allopregnanolone pathway. Front Neuroendocrinol. 2019;55:100789.

73. Reddy DS. Neurosteroids. Endogenous role in the human brain and therapeutic potentials. Prog Brain Res. 2010;186:113-137.

74. Wong P, et al. Pregnenolone rescues schizophrenia-like behavior in dopamine transporter knockout mice. PLoS One. 2012;7(12):e51455.

75. MacKenzie G, Maguire J. Neurosteroids and GABAergic signaling in health and disease. Biomol Concepts. 2013;4(1):29-42.

76. Gunn BG, et al. GABAA receptor-acting neurosteroids: a role in the development and regulation of the stress response. Front Neuroendocrinol. 2015;36:28-48.

77. Purdy RH, et al. Stress-induced elevations of $\gamma$-aminobutyric acid type a receptor-active steroids in the rat brain. Proc Natl Acad Sci US A.
1991;88(10):4553-4557.

78. Barbaccia ML, et al. Stress and neuroactive steroids. Int Rev Neurobiol. 2001;46:243-272.

79. Hu AC, et al. The efficacy and use of finasteride in women: a systematic review. Int J Dermatol. 2019;58(7):759-776.

80. Hojo Y, Kawato S. Neurosteroids in adult hippocampus of male and female rodents: biosynthesis and actions of sex steroids. Front Endocrinol (Lausanne). 2018;9:183.

81. Frau R, Bortolato M. Repurposing steroidogenesis inhibitors for the therapy of neuropsychiatric disorders: promises and caveats. Neuropharmacology. 2019;147:55-65.

82. Traish AM, et al. Adverse effects of $5 \alpha$-reductase inhibitors: what do we know, don't know, and need to know? Rev Endocr Metab Disord. 2015;16(3):177-198.

83. Andy G, et al. Controversies in the treatment of androgenetic alopecia: the history of finasteride. Dermatol Ther. 2019;32(2):e12647.

84. Mosher LJ, et al. Allopregnanolone mediates the exacerbation of Tourette-like responses by acute stress in mouse models. Sci Rep. 2017;7(1):1184-1189.

85. Bortolato M, et al. Antipsychotic-like properties of 5-alpha-reductase inhibitors. Neuropsychopharmacology. 2008;33(13):3146-3156.

86. Muroni A, et al. A preliminary study of finasteride in Tourette syndrome. Mov Disord. 2011;26(11):2146-2147.

87. Steiner JF. Clinical pharmacokinetics and pharmacodynamics of finasteride. Clin Pharmacokinet. 1996;30(1):16-27.

88. Gaikwad NW. Ultra performance liquid chromatography-tandem mass spectrometry method for profiling of steroid metabolome in human tissue. Anal Chem. 2013;85(10):4951-4960.

89. Nguyen TV, et al. The steroid metabolome in women with premenstrual dysphoric disorder during $\mathrm{GnRH}$ agonist-induced ovarian suppression: effects of estradiol and progesterone addback. Transl Psychiatry. 2017;7(8):e1193.

90. Gaikwad NW, inventor; Kilpatrick Townsend and Stockton LLP, assignee. Methods for comprehensive profiling of steroid metabolome. International patent WO2015161078 (A1). October 22, 2015.

91. Mavrikaki M, et al. Oxycodone self-administration in male and female rats. Psychopharmacology (Berl). 2017;234(6):977-987.

92. Shaham Y, et al. Effect of stress on oral fentanyl consumption in rats in an operant self-administration paradigm. Pharmacol Biochem Behav. 1993;46(2):315-322. 